\title{
RAMIFIED RECTILINEAR POLYGONS: COORDINATIZATION BY DENDRONS
}

\author{
HANS-JÜRGEN BANDELT, VICTOR CHEPOI, AND DAVID EPPSTEIN
}

\begin{abstract}
Simple rectilinear polygons (i.e. rectilinear polygons without holes or cutpoints) can be regarded as finite rectangular cell complexes coordinatized by two finite dendrons. The intrinsic $l_{1}$-metric is thus inherited from the product of the two finite dendrons via an isometric embedding. The rectangular cell complexes that share this same embedding property are called ramified rectilinear polygons. The links of vertices in these cell complexes may be arbitrary bipartite graphs, in contrast to simple rectilinear polygons where the links of points are either 4-cycles or paths of length at most 3. Ramified rectilinear polygons are particular instances of rectangular complexes obtained from cube-free median graphs, or equivalently simply connected rectangular complexes with triangle-free links. The underlying graphs of finite ramified rectilinear polygons can be recognized among graphs in linear time by a Lexicographic Breadth-First-Search. Whereas the symmetry of a simple rectilinear polygon is very restricted (with automorphism group being a subgroup of the dihedral group $D_{4}$ ), ramified rectilinear polygons are universal: every finite group is the automorphism group of some ramified rectilinear polygon.
\end{abstract}

\section{INTRODUCTION}

Polygons endowed with a metric are fundamental objects in computational and distance geometry. Computation of the geodesic distance between two query points of a polygon $P$ (that is, the length of the shortest path within $P$ that connects the two points) is required in a variety of algorithmic problems motivated by applications in robot motion, plant and facility layout, urban transportation, and wire layout; for a survey, see [32. To answer such queries efficiently, the polygon $P$ may be subdivided into simple pieces such as triangles, rectangles or trapezoids at the preprocessing stage. If $P$ is a simple polygon (bounded by a polygonal Jordan curve), then the dual graph of this subdivision will generally be a tree $T(P)$. Given a pair of points $s$ and $t$, the cells of the subdivision containing $s$ and $t$ may be computed using point-location methods [17]. On the other hand, the dual graph of the subdivision gives a rough idea of the global location of $s$ and $t$ in the polygon $P$, yielding essential information for computing the exact geodesic distance between $s$ and $t$. In particular, if $P$ is simple, and the boundaries between cells of the subdivision are straight line segments, then any shortest $(s, t)$-path traverses the cells of the subdivision along the unique path of $T(P)$ that connects the cells containing $s$ and $t$. Therefore the subdivision of $P$ can be viewed as a kind of coordinatization of the points of $P$.

A simple rectilinear polygon (also called an orthogonal polygon) is a simple polygon $P$ for which all sides (boundary segments) are parallel to the coordinate axes; we view such 


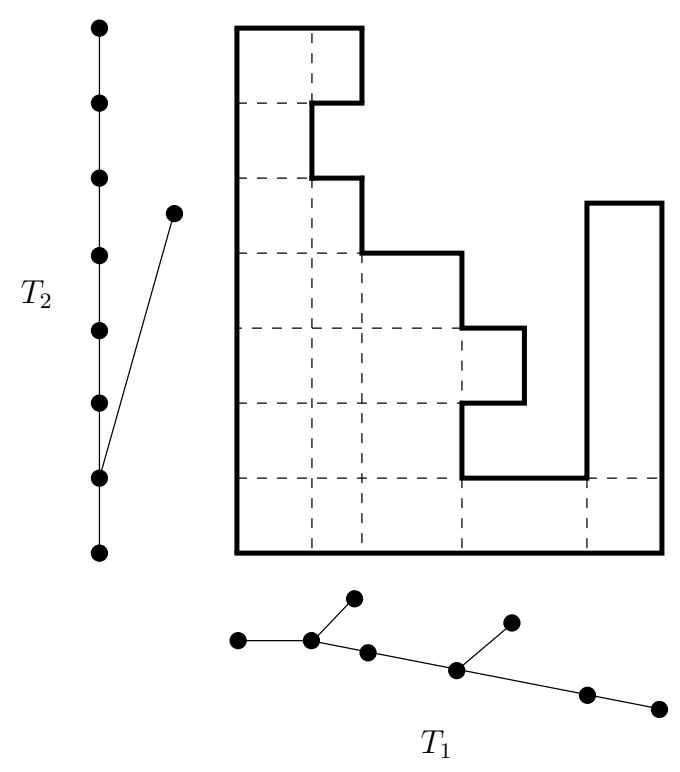

Figure 1. A simple rectilinear polygon $P$ and its grid lines giving rise to the coordinate tree networks $T_{1}$ and $T_{2}$.

polygons as being topologically closed (that is, they include their boundary segments) and endowed with the $l_{1}$-metric. For simple rectilinear polygons the idea of coordinatization by trees can be made more precise, as we describe below, so that geodesic distances can be directly computed from the tree-coordinates of the points. However, this more precise coordinatization involves two trees rather than one, using a subdivision of the polygon into rectangles that does not have a single tree as its dual.

A corner of $P$ is a point on the boundary of $P$ incident with two (perpendicular) sides. We call a horizontal or vertical axis-parallel line segment a line of $P$ if it lies entirely in $P$ and is maximal with respect to inclusion. A grid line of $P$ is any line of $P$ passing through some corner of $P$. The grid network $N(P)$ has all points in which horizontal and vertical grid lines intersect as its vertices (thus including all corners); in computational geometry terminology, it is the arrangement formed by the grid lines. Two vertices $u$ and $v$ of $N(P)$ are adjacent exactly when they are consecutive on a common line, so that $N(P)$ forms a planar graph; the edge $u v$ of $N(P)$ is then weighted by the length of the line segment between $u$ and $v$. The shortest-path metric $d_{N}$ of the grid network $N(P)$ can be extended to a metric $d_{P}$ of the entire simple polygon $P$ in the following way. For any two distinct points $s$ and $t$ of $P$ augment the set of grid lines by the lines of $P$ that pass through $s$ or $t$. Define the expanded grid network $N_{s, t}(P)$ with respect to this enlarged set of distinguished lines, so that $d_{P}(s, t)$ is the distance in the network $N_{s, t}(P)$. A shortest path in $N_{s, t}(P)$ joining $s$ and $t$ can be turned into a rectilinear path $\pi_{s t}$ of $P$ having length $d_{P}(s, t)$ by replacing each edge of $N_{s, t}(P)$ by the corresponding axis-parallel line segment in $P$. In general, a rectilinear path $\pi$ of $P$ is any polygonal chain consisting of axis-parallel segments lying inside $P$, and its length is the sum 
of lengths of the constituent segments of $P$. It is easy to see that $d_{P}(s, t)$ equals the intrinsic $l_{1}$-distance (or geodesic distance) of $P$ between $s$ and $t$, that is, the minimum length of a rectilinear path connecting $s$ and $t$.

Partitioning the set of grid lines into the horizontal and the vertical lines gives rise to two tree networks, $T_{1}$ and $T_{2}$; see Fig. 1 for an example. Namely, the vertices of $T_{1}$ are the vertical grid lines, where two lines are adjacent exactly when they support opposite edges of some 4-cycle of $N(P)$. The weight of the respective edge of $T_{1}$ is then the horizontal distance between the two vertical lines, which is the length of the perpendicular edges of the supported 4-cycle of $N(P)$. Similarly, $T_{2}$ is defined in terms of the horizontal grid lines. Every vertex $x$ of $N(P)$ thus receives two coordinates $x_{1}$ and $x_{2}$ in $T_{1}$ and $T_{2}$, respectively, such that the mapping $x \mapsto\left(x_{1}, x_{2}\right)$ constitutes an isometric embedding of the network $N(P)$ into the Cartesian product $T_{1} \square T_{2}$ of the tree networks $T_{1}$ and $T_{2}$.

If every edge of $T_{1}$ and $T_{2}$, respectively, is replaced by a segment of the real line having the same length, one obtains finite dendrons $D_{1}$ and $D_{2}$ (for definitions, see Section 2). The points of $D_{1}$ are then in one-to-one correspondence with the vertical lines of $P$, and the points of $D_{2}$, mutatis mutandis, with the horizontal lines. The isometric embedding of $N(P)$ into the Cartesian product $T_{1} \square T_{2}$ therefore lifts to an isometric embedding of $P$ into the product space $D_{1} \times D_{2}$. After linear time preprocessing, distance queries in trees and dendrons may be performed in constant time using lowest common ancestor algorithms [1,2, 26]. Since the $l_{1}$ geodesic distance between any two points in $P$ is just the sum of distances between the corresponding points in the two factors of the Cartesian product, this shows that distance computations in simple rectilinear polygons are reduced to finding the coordinates of the query points in the factors.

This coordinatization of simple rectilinear polygons motivates us to consider and investigate the rectilinear complexes endowed with the intrinsic $l_{1}$-metric that have isometric embeddings into Cartesian products of two dendrons. We will call such objects "ramified rectilinear polygons." A straightforward inductive argument shows that every tree with more than one node can occur in the coordinatization of a simple rectilinear polygon. However, not every pair of trees may occur in combination; for instance, if $T_{1}$ is the star $K_{1, k}$, then $T_{2}$ must have at least $\max (k+1,2 k-2)$ nodes, whereas this constraint does not apply in the ramified case. Therefore, ramified rectilinear polygons are a strict generalization of simple rectilinear polygons.

In this paper we characterize the ramified rectilinear polygons and their underlying networks (the networks playing the same role for these complexes as the network $N(P)$ played for the simple rectilinear polygon $P$ ); see Section 3 for details of this characterization. The underlying networks of ramified rectilinear polygons are exactly the networks that can be isometrically embedded into the Cartesian product of two tree networks; our characterization provides a simple linear time algorithm for their recognition. Note, however, that for every $k \geq 3$ the problem of recognizing the graphs isometrically embeddable into the Cartesian product of $k$ trees is NP-complete [8]. For a survey of other relationships between the metric geometry of complexes and that of graphs, see 6 . More generally, in a subsequent paper, we 
investigate the complete Menger-convex metric spaces that can be isometrically embedded into the products of two dendrons. We call such spaces "partial double dendrons."

\section{THE DISCRETE AND GEOMETRIC FRAMEWORKS}

2.1. Metric spaces. Let $(X, d)$ be a metric space. An arc joining two points $x, y$ of $X$ is a homeomorphic map $\gamma:[0, l] \rightarrow X$ such that $\gamma(0)=x$ and $\gamma(l)=y$. Then $(X, d)$ is called arc-connected if any two points of $X$ can be joined by an arc. Given $x \in X$ and $r>0$, the open ball $\{y \in X: d(x, y)<r\}$ of radius $r$ centered at $x$ is denoted by $B^{\circ}(x, r)$, and the corresponding closed ball $\{y \in X: d(x, y) \leq r\}$ is denoted by $B(x, r)$. The interval between two points $x, y$ of $X$ is the set $I(x, y)=\{z \in X: d(x, y)=d(x, z)+d(z, y)\}$; for example, in Euclidean spaces, the interval $I(x, y)$ is the closed line segment having $x$ and $y$ as its endpoints. The space $(X, d)$ is called Menger-convex if for any two distinct points $x, y \in X$ there exists a point $z \in I(x, y)$ different from $x, y$. If, in addition to being Menger-convex, $(X, d)$ is (Cauchy-)complete, it follows that for any two points $x, y$ and every $0 \leq t \leq 1$ there exists a point $z \in X$ such that $d(x, z)=t \cdot d(x, y)$ and $d(z, y)=(1-t) \cdot d(x, y)$ [1]. A geodesic joining two points $x$ and $y$ from $X$ is the image of a (continuous) map $\gamma$ from a line segment $[0, l] \subset \mathbb{R}$ to $X$ such that $\gamma(0)=x, \gamma(l)=y$ and $d\left(\gamma(t), \gamma\left(t^{\prime}\right)\right)=\left|t-t^{\prime}\right|$ for all $t, t^{\prime} \in[0, l]$. The space $(X, d)$ is said to be geodesic if every pair of points $x, y \in X$ is joined by a geodesic (which is necessarily included in $I(x, y)$ ) [13, 35]. Since every geodesic is an arc, a geodesic space is arc-connected. Every complete Menger-convex metric space is geodesic [11,31.

A dendron $D$ is a complete Menger-convex metric space in which any two distinct points $x, y$ can be joined by a unique arc $\gamma(x, y)$. Note that $\gamma(x, y)$ is the unique geodesic joining the points $x, y$. In fact, $\gamma(x, y)$ coincides with $I(x, y)$. To see this, assume for a contradiction that there exists a point $z$ that is in $I(x, y)$ but outside $\gamma(x, y)$. Let $\gamma(x, z)$ and $\gamma(y, z)$ be the two geodesics connecting the points $x$ and $y$ with $z$. The choice of $z$ in $I(x, y)$ implies that $\gamma(x, z) \cap \gamma(y, z)=\{z\}$. Therefore concatenating $\gamma(x, z)$ and $\gamma(z, y)$ will lead to an arc joining $x$ and $y$ different from $\gamma(x, y)$, contradicting the assumption that arcs are unique. This establishes that indeed $\gamma(x, y)=I(x, y)$. A leaf of a dendron $D$ is a point whose removal does not separate any pair of points and a ramification point is a point whose removal creates at least three arc-connected components. The leaves and ramifications points are called the vertices of $D$. A finite dendron [12] is a compact dendron with a finite number of vertices. A finite dendron $D$ may be formed from a finite tree $T$ by replacing each edge of $T$ by a line segment; the vertices of $D$ correspond to vertices of $T$, but $T$ may have additional degree-two vertices that do not correspond to vertices in $D$.

The (direct or Cartesian) product of two metric spaces $\left(X_{1}, d_{1}\right)$ and $\left(X_{2}, d_{2}\right)$ is the set $X_{1} \times X_{2}$ endowed with the metric $d\left(\left(x_{1}, x_{2}\right),\left(y_{1}, y_{2}\right)\right)=d_{1}\left(x_{1}, y_{1}\right)+d_{2}\left(x_{2}, y_{2}\right)$. A function $f: X \rightarrow X^{\prime}$ between two metric spaces $(X, d)$ and $\left(X^{\prime}, d^{\prime}\right)$ is an isometric embedding of $X$ into $X^{\prime}$ when $d^{\prime}(f(x), f(y))=d(x, y)$ for any $x, y \in X$. In this case $Y:=f(X)$ is called an (isometric) subspace of $X^{\prime}$. We also refer to the product $D=D_{1} \times D_{2}$ of two dendrons $D_{1}, D_{2}$ as a double dendron, for short. A partial double dendron is then a complete Menger-convex isometric subspace of a double dendron. 
A subspace $Y$ of a metric space $(X, d)$ is gated if for every point $x \in X$ there exists a (unique) point $x^{\prime} \in Y$, the gate of $x$ in $Y$, such that $d(x, y)=d\left(x, x^{\prime}\right)+d\left(x^{\prime}, y\right)$ for all $y \in Y$ [18]. The intersection of two gated subspaces is a gated subspace again. $(X, d)$ is said to be a gated amalgam of two smaller spaces $\left(Y, d_{Y}\right)$ and $\left(Z, d_{Z}\right)$ along a common nonempty gated subspace $Y \cap Z$ if $X=Y \cup Z$ and $d$ extends $d_{Y}$ and $d_{Z}$ with

$$
d(y, z):=d_{Y}\left(y, y^{\prime}\right)+d_{Z}\left(y^{\prime}, z\right)=d_{Y}\left(y, z^{\prime}\right)+d_{Z}\left(z^{\prime}, z\right) \text { for all } y \in Y \text { and } z \in Z,
$$

where $x \mapsto x^{\prime}$ denotes the gate map from $Y$ as well as from $Z$ to $Y \cap Z$. A particular instance of this kind of amalgamation is given when $Z$ is the product of some subspace $U$ of $Y$ with the real interval $[0, \lambda]$ of length $\lambda>0$ where the fiber $U \times\{0\}$ gets identified with $U$. The gated amalgam of $Y$ and the product $Z=U \times[0, \lambda]$ along $U=Y \cap Z$ is then referred to as the gated expansion of $Y$ by $[0, \lambda]$ along $U$. Gated subspaces are necessarily interval-convex, where a subspace $Y$ of $X$ is called interval-convex if $I(x, y) \subseteq Y$ for any $x, y \in Y$. The convex hull $\operatorname{conv}(Z)$ of $Z \subset X$ is the smallest convex subspace containing $Z$. A half-space $H$ of $X$ is a convex subspace with a convex complement. The partition $\{H, X \backslash H\}$ is called a convex split of $X$.

Let $(X, d)$ be a metric space and $x, y, z \in X$. We often use "median" sets of the type $m(x, y, z)=I(x, y) \cap I(y, z) \cap I(z, x)$. If $m(x, y, z)$ is a singleton for all $x, y, z \in X$, then the space $X$ is called median [39] and we usually refer to $m(x, y, z)$ as to the median of $x, y, z$ (here we do not distinguish between the singleton and the corresponding point). Dendrons are median spaces. Indeed, given three points $x, y, z$ of a dendron $D$, the union $\gamma(x, y) \cup \gamma(x, z)$ contains an arc between $y$ and $z$ which is composed of two subarcs of $\gamma(x, y)$ and $\gamma(x, z)$ intersecting in a single point $m$. By uniqueness of arcs, the composed arc coincides with the geodesic $\gamma(y, z)$ [31]. Therefore, as geodesics are intervals, $m$ is the unique median of $x, y, z$. The product of any two median spaces is evidently median, and therefore products of dendrons are median. But in general, an isometric subspace $Y$ of a median space $X$ need not be median, unless it is median-stable, that is, $m(x, y, z) \in Y$ for all $x, y, z \in Y$.

A geodesic triangle $\Delta\left(x_{1}, x_{2}, x_{3}\right)$ in a geodesic metric space $(X, d)$ consists of three distinct points in $X$ (the vertices of $\Delta$ ) and a geodesic between each pair of vertices (the sides of $\Delta$ ). A comparison triangle for $\Delta\left(x_{1}, x_{2}, x_{3}\right)$ is a triangle $\Delta\left(x_{1}^{\prime}, x_{2}^{\prime}, x_{3}^{\prime}\right)$ in the Euclidean plane $\mathbb{E}^{2}$ such that $d_{\mathbb{E}^{2}}\left(x_{i}^{\prime}, x_{j}^{\prime}\right)=d\left(x_{i}, x_{j}\right)$ for $i, j \in\{1,2,3\}$. A geodesic metric space $(X, d)$ is defined to be a CAT(0) space [25] if all geodesic triangles $\Delta\left(x_{1}, x_{2}, x_{3}\right)$ of $X$ satisfy the comparison axiom of Cartan-Alexandrov-Toponogov:

If $y$ is a point on the side of $\Delta\left(x_{1}, x_{2}, x_{3}\right)$ with vertices $x_{1}$ and $x_{2}$ and $y^{\prime}$ is the unique point on the line segment $\left[x_{1}^{\prime}, x_{2}^{\prime}\right]$ of the comparison triangle $\Delta\left(x_{1}^{\prime}, x_{2}^{\prime}, x_{3}^{\prime}\right)$ such that $d_{\mathbb{E}^{2}}\left(x_{i}^{\prime}, y^{\prime}\right)=$ $d\left(x_{i}, y\right)$ for $i=1,2$, then $d\left(x_{3}, y\right) \leq d_{\mathbb{E}^{2}}\left(x_{3}^{\prime}, y^{\prime}\right)$.

This simple axiom turns out to be very powerful, because CAT $(0)$ spaces can be characterized in several natural ways (for a full account of this theory consult the book [13]). CAT(0) spaces play a vital role in modern combinatorial group theory, where various versions of hyperbolicity are related to group-theoretic properties [23, 25]; many arguments in this area have a strong metric graph-theoretic flavor. A geodesic metric space $(X, d)$ is $\mathrm{CAT}(0)$ if and only if any 
two points of this space can be joined by a unique geodesic. CAT(0) is also equivalent to convexity of the function $f:[0,1] \rightarrow X$ given by $f(t)=d(\alpha(t), \beta(t))$, for any geodesics $\alpha$ and $\beta$ (which is further equivalent to convexity of the neighborhoods of convex sets). This implies that $\operatorname{CAT}(0)$ spaces are contractible.

2.2. Graphs and networks. Connected undirected graphs $G=(V, E)$ endowed with the graph metric $d_{G}$ defined by

$$
d_{G}(x, y)=\text { the length of a shortest path between } x \text { and } y
$$

are basic examples of discrete metric spaces. An induced subgraph $G^{\prime}=\left(V^{\prime}, E^{\prime}\right)$ of $G$ is a subset of vertices with the induced edge-relation, and it is an isometric subgraph if the distances between any two vertices of $V^{\prime}$ in $G$ and $G^{\prime}$ coincide. These notions naturally carry over to networks, i.e. graphs with weighted edges.

A graph $G$ is a median graph if $\left(V, d_{G}\right)$ is a median space [3]. Discrete median spaces, in general, can be regarded as median networks: a median network is a median graph with weighted edges such that opposite edges in any 4-cycle have the same length [4]. Typical examples of median graphs are hypercubes (weak Cartesian powers of $K_{2}$ ), trees, and squaregraphs (i.e., plane graphs in which all inner faces are 4-cycles and all inner vertices have degrees $\geq 4[7,[15])$. Finite median graphs are exactly the graphs which are obtained from finite hypercubes by applying successive gated amalgamations [29, 38]. It is well known [8,33] that median graphs isometrically embed into hypercubes and therefore into Cartesian products of trees. A median graph contains an isometric 6-cycle if and only if it contains an induced cube $Q_{3}$, for the two remaining cube vertices are forced to exist as medians of alternating subsets of vertices from the 6-cycle. Median graphs or networks not containing any induced cube (or cube network, respectively) are called cube-free.

The isometric embedding of a median graph $G$ into a (smallest) hypercube coincides with the so-called canonical embedding, which is determined by the Djoković-Winkler relation $\Theta$ on the edge set of $G$ : two edges $u v$ and $w x$ are $\Theta$-related exactly when

$$
d_{G}(u, w)+d_{G}(v, x) \neq d_{G}(u, x)+d_{G}(v, w) ;
$$

see [21,27]. For a median graph this relation is transitive and hence an equivalence relation. It is the transitive closure of the "opposite" relation of edges on 4-cycles: in fact, any two $\Theta$-related edges can be connected by a ladder (viz., the Cartesian product of a path with $\left.K_{2}\right)$, and the block of all edges $\Theta$-related to some edge $u v$ constitute a cutset $\Theta(u v)$ of the median graph, which determines one factor of the canonical hypercube [33, 34]. The cutset $\Theta(u v)$ defines a convex split $\sigma(u v)=\{W(u, v), W(v, u)\}$ of $G$ [34, 39, where $W(u, v)=\{x \in$ $X: d(u, x)<d(v, x)\}$ and $W(v, u)=V-W(u, v)$. Conversely, for every convex split of a median graph $G$ there exists at least one edge $x y$ such that $\{W(x, y), W(y, x)\}$ is the given split. Two convex splits $\sigma_{1}=\left\{A_{1}, B_{1}\right\}$ and $\sigma_{2}=\left\{A_{2}, B_{2}\right\}$ of $G$ are said to be incompatible if all four intersections $A_{1} \cap A_{2}, A_{1} \cap B_{2}, B_{1} \cap A_{2}$, and $B_{1} \cap B_{2}$ are non-empty, and are called compatible otherwise. The incompatibility graph $\operatorname{Inc}(G)$ of $G$ has the convex splits of $G$ as vertices and pairs of incompatible convex splits as edges. 


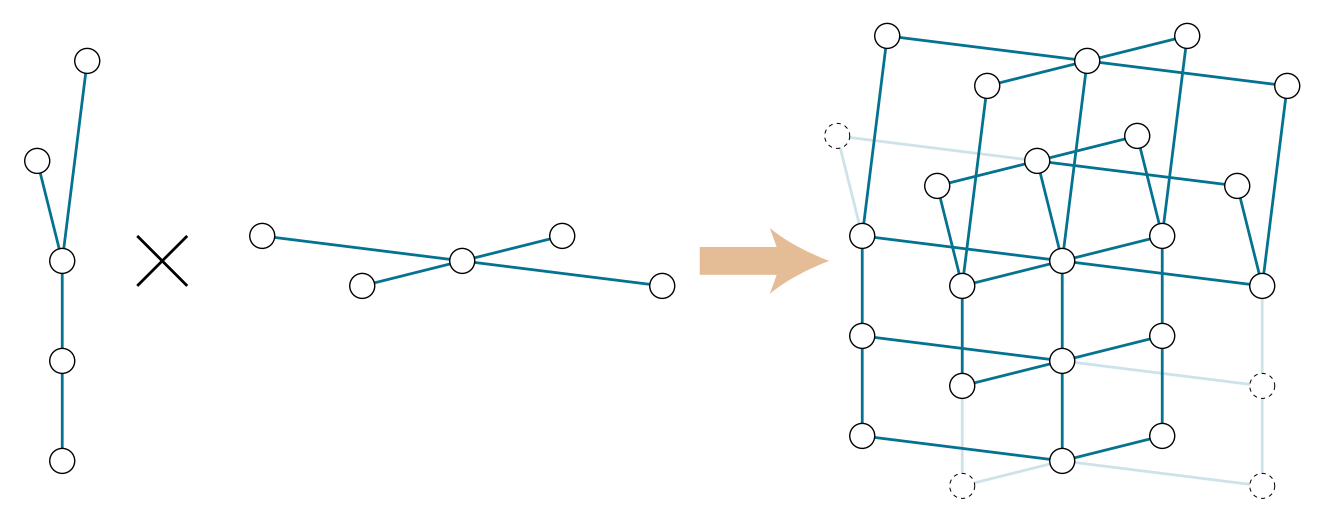

Figure 2. An isometric subgraph of the Cartesian product of two trees

The convex hull conv $(X)$ of any finite set $X$ of vertices in an infinite median graph $G$ is known to form a finite median subgraph of $G$ [39]. Therefore $G$ is a directed union of its finite convex subgraphs. This will be used in many arguments for transferring a result from finite to infinite median graphs: in particular, any property that can be expressed in terms of finitely many convex sets or splits carries over from the finite to the infinite case.

2.3. Rectangular complexes and ramified rectilinear polygons. In this subsection we recall and specify some terminology about cell complexes; for general notions, see [13, 39]. A rectangular complex $\mathcal{K}$ is a 2-dimensional cell complex $\mathcal{K}$ whose 2-cells are isometric to axis-parallel rectangles of the $l_{1}$-plane; unlike some related work [20] we require that the 1-cells of the complex be complete edges of these rectangles, so the nonempty intersection of any two rectangles must either be a vertex or an edge of both of them. If all 1-cells of $\mathcal{K}$ have equal length, then we call $\mathcal{K}$ a square complex; in this case we may assume without loss of generality that the squares of the complex are all unit squares. Square complexes are the 2-dimensional instances of cubical complexes, viz. the cell complexes (where cells have finite dimension) in which every cell of dimension $k$ is isometric to the unit cube of $\mathbb{R}^{k}$. It is not stipulated here that the cell complexes have only finitely many cells, but, as in [13], we require that the complex contains only finitely many isometry types of rectangular cells.

The 0 -dimensional faces of a rectangular complex $\mathcal{K}$ are called its vertices, forming the vertex set $V(\mathcal{K})$ of $\mathcal{K}$. The underlying network $N(\mathcal{K})$ of $\mathcal{K}$ then represents the 1 -dimensional faces by weighted edges of the same length. Disregarding edge lengths (by adopting unit lengths) one obtains the underlying graph $G(\mathcal{K})=(V(\mathcal{K}), E(\mathcal{K}))$. In the case of a simple rectilinear polygon $P$, the cells of the associated rectangular complex $\mathcal{K}(P)$ are determined by a pair of adjacent vertical grid lines together with a pair of adjacent horizontal grid lines. Then the underlying network of $\mathcal{K}(P)$ is the grid network $N(P)$ of $P$. Conversely, from any graph $G$ or network $N$ one can derive a cube or box complex $|G|$ or $|N|$ by replacing all cuboids, i.e. subgraphs (or subnetworks) of $G$ (or $N$ ) isomorphic to (edge-weighted) cubes of any dimensions (vertices, edges, induced 4-cycles, etc.) by solid boxes. The complexes $|G|$ and $|N|$ are referred to as the geometric realization of $G$ and $N$, respectively. In particular, 

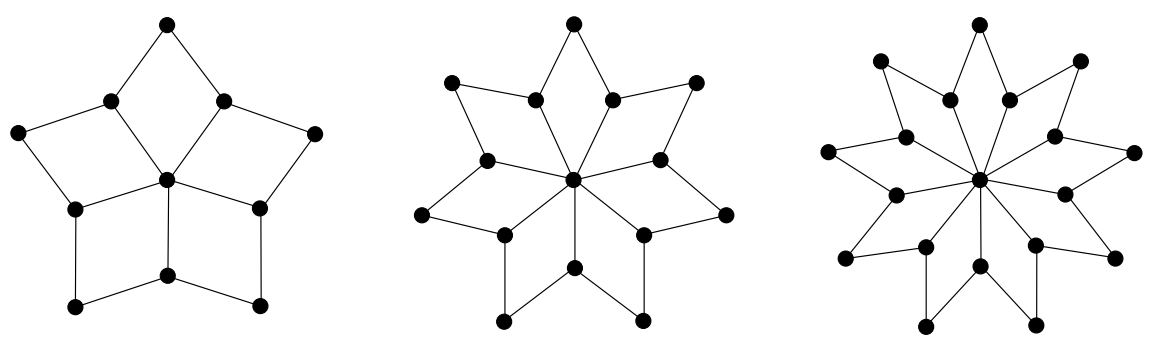

FiguRE 3. The first three odd cogwheels

$\mathcal{K}(P)=|N(P)|$ for every simple rectilinear polygon $P$. More generally, median networks give rise to particularly interesting cubical complexes of higher dimensions.

A cell complex $\mathcal{K}$ is called simply connected if it is connected and every continuous mapping of the 1 -dimensional sphere $\mathbb{S}^{1}$ into $\mathcal{K}$ can be extended to a continuous mapping of the disk $\mathbb{D}^{2}$ with boundary $\mathbb{S}^{1}$ into $\mathcal{K}$. The link of a vertex $x$ in $\mathcal{K}$ is the graph $\operatorname{Link}(x)$ whose vertices are the 1-cells containing $x$ and where two 1-cells are adjacent if and only if they are contained in a common 2-cell (see 13 for the notion of link in general polyhedral complexes). The link graph $\operatorname{Link}(\mathcal{K})$ of $\mathcal{K}$ is then the union of the graphs $\operatorname{Link}(x)$ for all vertices $x$ of $\mathcal{K}$.

A rectangular complex $\mathcal{K}$ can be endowed with several intrinsic metrics 13 transforming $\mathcal{K}$ into a complete geodesic space. Suppose that inside every 2-cell of $\mathcal{K}$ the distance is measured according to an $l_{1^{-}}, l_{2^{-}}$, or $l_{\infty^{-}}$metric (or, more generally, according to any $l_{p^{-}}$ metric). The intrinsic $l_{1^{-}}$or $l_{2}$-metric of $\mathcal{K}$ is defined by assuming that the distance between two points $x, y \in \mathcal{K}$ equals the infimum of the lengths of the paths joining them. Here a path in $\mathcal{K}$ from $x$ to $y$ is a sequence $P$ of points $x=x_{0}, x_{1} \ldots x_{m-1}, x_{m}=y$ such that for each $i=0, \ldots, m-1$ there exists a 2 -cell $R_{i}$ containing $x_{i}$ and $x_{i+1}$; the length of $P$ is $l(P)=\sum_{i=0}^{m-1} d\left(x_{i}, x_{i+1}\right)$, where $d\left(x_{i}, x_{i+1}\right)$ is computed inside $R_{i}$ according to the respective metric. A ramified rectilinear polygon is a finite rectangular complex $\mathcal{K}$ endowed with the intrinsic $l_{1}$-metric which embeds isometrically into the product of two finite dendrons.

\section{MAin Results}

In this section we formulate the main results of the paper; in particular, we provide geometric and combinatorial characterizations of ramified rectilinear polygons, along with their underlying graphs and, more generally, arbitrary partial double dendrons. We start with a characterization of graphs $G$ which are isometrically embeddable in the Cartesian product of two trees (see Fig. 2 for an example). We call such graphs partial double trees. Such graphs are bipartite and cube-free, that is, the cube $Q_{3}$ does not occur as an induced subgraph. For a graph $F$ denote the simplex graph [8] of $F$ by $\kappa(F)$ : it has the simplices (or cliques) of $F$ as its vertices, and two vertices in $\kappa(F)$ are connected by an edge when their simplices differ by the presence or absence of exactly one vertex of $F$. The cube $Q_{3}$ is isomorphic to $\kappa\left(C_{3}\right)$, and when $F=C_{n}$ is a cycle with $n>3$ vertices, then $\kappa(F)$ is called a cogwheel [30] or bipartite wheel [5]. A median graph is cube-free exactly when it does not contain a subgraph 
isomorphic to $Q_{3}-v$, the cube minus a vertex. There exist, however, cube-free median graphs that are not partial double trees: each odd cogwheel $\kappa\left(C_{2 k+1}\right)$ for $k \geq 2$ (Fig. 3 ) is a cubefree isometric subgraph of $Q_{2 k+1}$, yet the least number $\tau(G)$ of tree factors in a Cartesian representation of the graph $G=\kappa\left(C_{2 k+1}\right)$ equals 3. The graphs $\kappa\left(C_{2 k+1}\right)$ play a key role in our characterization, as they signify odd cycles in the links of vertices in the complex $|G|$.

Theorem 1. For a connected graph $G$ the following conditions are equivalent:

(i) $G$ is a partial double tree;

(ii) $G$ is a median subgraph of the Cartesian product of two trees;

(iii) $G$ is a median graph such that $\operatorname{Inc}(G)$ is bipartite;

(iv) $G$ is a median graph such that $\operatorname{Link}(|G|)$ is bipartite;

(v) $G$ is a median graph such that $\operatorname{Link}(x)$ is bipartite for all vertices $x$ of $G$;

(vi) $G$ is a median graph that does not contain $Q_{3}-v$ nor any $\kappa\left(C_{2 k+1}\right)(k \geq 2)$ as an isometric subgraph;

(vii) $G$ is an isometric subgraph of a hypercube such that $C_{2 k+2}$ and $\kappa\left(C_{2 k+1}\right)(k \geq 2)$ are not isometric subgraphs of $G$;

(viii) $C_{3}, K_{2,3}, C_{k+3}$, and $\kappa\left(C_{2 k+1}\right)(k \geq 2)$ are not isometric subgraphs of $G$.

In this theorem, the isometric embedding of a partial double dendron into the Cartesian product of two trees is in general not unique, even when the two projections are onto (that is, the two trees are minimal). Uniqueness of the embedding relative to permutation and automorphisms of the two trees is achieved under 2-connectivity:

Corollary 1. For a connected graph $G$ with at least three vertices, the following conditions are equivalent:

(i) $G$ is a partial double tree such that $G$ has a unique isometric embedding into the Cartesian product of two trees relative to minimality, permutations and automorphisms of the two trees;

(ii) $G$ is 2-connected median subgraph of the Cartesian product of two trees;

(iii) $G$ is a median graph such that $\operatorname{Inc}(G)$ is bipartite and connected;

(iv) $G$ is a median graph such that $\operatorname{Link}(x)$ is bipartite and connected for all vertices $x$ of $G$;

(v) $G$ is a median graph such that $\operatorname{Link}(G)$ is bipartite and connected.

The characterization of partial double trees provided by Theorem 1(v) can be used to recognize partial double trees in polynomial time. As we will show below, this task can be implemented in linear time.

Theorem 2. For a finite graph $G=(V, E)$, one can decide in linear time $O(|V|+|E|)$ whether $G$ is a partial double tree.

The ease of recognizing partial double trees, as codified by Proposition 2, stands in contrast with the difficulty of recognizing the generalization of partial double trees to cube-free median graphs. If $G$ is a triangle-free graph, then the graph formed from a graph $G$ by adding a 
new vertex adjacent to every vertex in $G$, and by subdividing every edge in $G$, is the simplex graph of $G$ and is a cube-free median graph. But if $G$ is not triangle-free, then the graph derived from it in this way is not a median graph. Hence, recognition of cube-free median graphs is no easier than (and is in fact equivalent in computational difficulty to) recognition of triangle-free graphs, for which no linear time algorithms are known [28].

We continue with a characterization of the rectangular complexes that arise from cubefree median graphs. These complexes are more general than ramified rectilinear polygons; nevertheless they share one significant property with the latter: equipped with the $l_{1}$-metric they define metric spaces that are median.

Theorem 3. For a rectangular complex $\mathcal{K}$ the following conditions are equivalent:

(i) the underlying graph $G(\mathcal{K})$ of $\mathcal{K}$ is a cube-free median graph;

(ii) $\mathcal{K}$ equipped with the intrinsic $l_{1}$-metric $d$ is median;

(iii) $\mathcal{K}$ equipped with the intrinsic $l_{2}$-metric is $\mathrm{CAT}(0)$;

(iv) $\mathcal{K}$ is simply connected and for every vertex $x \in V(\mathcal{K})$, the graph $\operatorname{Link}(x)$ is $C_{3}$-free.

If any of these conditions holds for the rectangular complex $\mathcal{K}$, then the metric space $\mathcal{K}$ coincides with the geometric realization $|N(\mathcal{K})|$ of its network.

The next result characterizes the ramified rectilinear polygons and the partial double dendrons. As a consequence, one obtains a characterization of all metric spaces isometrically embeddable into double dendrons.

Theorem 4. For a finite rectangular complex $\mathcal{K}$ equipped with the intrinsic $l_{1}$-metric $d$ the following conditions are equivalent:

(i) $\mathcal{K}$ is a ramified rectilinear polygon;

(ii) $\mathcal{K}$ is median and each vertex $x \in V(\mathcal{K})$ has some closed neighborhood $B(x, \epsilon)$ (where $\epsilon>0)$ that is a partial double dendron;

(iii) $\mathcal{K}$ is simply connected and each vertex $x \in V(\mathcal{K})$ has some closed neighborhood $B(x, \epsilon)$ (where $\epsilon>0$ ) that is a partial double dendron;

(iv) $\mathcal{K}$ is simply connected and for each vertex $x \in V(\mathcal{K})$, the graph $\operatorname{Link}(x)$ is bipartite;

(v) the underlying graph $G(\mathcal{K})$ of $\mathcal{K}$ is a partial double tree and $\mathcal{K}=|G(\mathcal{K})|$;

(vi) the metric space $(\mathcal{K}, d)$ can be obtained from a singleton space by a finite sequence of gated expansions by real intervals along gated dendrons supporting exclusively even cogfans;

(vii) the metric space $(\mathcal{K}, d)$ can be obtained from finitely many rectangles (equipped with the $l_{1}$-metric) via successive gated amalgamations along gated dendrons that in each point support cogfans in either part of the same parity exclusively.

We will finally show that median graphs are universal with respect to their automorphism groups. This is not surprising in view of Birkhoff's Theorem stating that every group is the automorphism group of some distributive lattice (and the close relationship between distributive lattices and median algebras [10]). What might, however, be surprising at first sight is the fact that the partial double trees are already universal. 
Theorem 5. Every group is the automorphism group aut $(G)$ of a 2-connected partial double tree $G$ of radius 2. If the group is finite, the graph can be taken to be finite. The automorphism group of $G$ is isomorphic to the group of isometries of the geometric realization $|G|$, which is a ramified rectilinear polygon without cutpoints in the case that $G$ is finite.

\section{Proof of Theorem 1 and Corollary 1}

We begin with a known characterization of the cube-free median graphs (see [5]): a connected graph is a cube-free median graph if and only if it does not contain $C_{3}, K_{2,3}$, or $C_{k+3}$ (for $k \geq 2$ ) as isometric subgraphs. Because the relation of being an isometric subgraph is transitive, this implies that an isometric subgraph of a cube-free median graph is itself median. Because trees are median graphs and the product operation preserves median graphs, the Cartesian product $H$ of any finite family of trees is a median graph; if the number of factors equals two, then $H$ is cube-free. Thus, the partial double trees are isometric subgraphs of cube-free median graphs, and are themselves median, giving us the equivalence (i) $\Longleftrightarrow(i i)$ of Theorem 1 .

The equivalence (ii) $\Longleftrightarrow$ (iii) of Theorem 1 is a particular case of a result of [8] establishing that a median graph $G$ embeds into the Cartesian product of $n$ trees if and only if $\operatorname{Inc}(G)$ is $n$-colorable. For each vertex $x$ of $G, \operatorname{Link}(x)$ is an induced subgraph of $\operatorname{Link}(|G|)$, therefore if $\operatorname{Link}(|G|)$ is bipartite, then all links of vertices are bipartite as well, inferring that (iv) $\Longrightarrow(\mathrm{v})$. Since a graph is bipartite exactly when it does not contain an isometric odd cycle, the condition (vi) is a reformulation of $(\mathrm{v})$, whence the equivalence $(\mathrm{v}) \Longleftrightarrow$ (vi) holds as well.

Note that every isometric subgraph of a hypercube is bipartite and does not contain any induced $K_{2,3}$. By combining this fact with the characterization of cube-free median graphs by forbidden isometric subgraphs and the characterization of cube-free median graphs as the median graphs without $Q_{3}-v$, we see immediately that the equivalences (vi) indeed hold. It remains to show that the conditions (ii), (iv), and (vi) are equivalent, as we do in the rest of this section. At the end of the section, an example will indicate that, in contrast to $(\mathrm{v})$, there does not exist a characterization of isometric subgraphs of products of three trees in terms of the links of their vertices.

Since the canonical embedding [24,27] of a median graph $G$ into a hypercube is governed by its transitive relation $\Theta$, which is the transitive hull of the relation formed by opposite edges from 4-cycles, every isometric embedding of $G$ into a Cartesian product of graphs can be expressed in terms of (improper) edge-coloring such that opposite edges in every 4-cycle are equally colored. With the factors of the corresponding Cartesian product being canonical images of $G$, this constitutes a subdirect representation of the associated median algebras. For the sake of minimizing the necessary background information, the next two lemmas provide a direct proof.

Lemma 1. A median graph $G$ is a subgraph of a Cartesian product of $n$ graphs $H_{i}$ if and only if the edges of $G$ may be (improperly) colored with $n$ colors in such a way that every two opposite edges of a 4-cycle of $G$ are assigned the same color. If the projection $\pi_{i}$ from 
$G$ to each factor $H_{i}$ is onto, then each factor $H_{i}$ must itself be a median graph, and a graph isomorphic to $H_{i}$ may be recovered by contracting every edge of $G$ that does not have color $i$.

Proof. First we observe that subgraphs of products have the edge colorings described in the lemma. To see this, consider any subgraph $G$ of the Cartesian product $H$ of graphs $H_{i}$ $(i=1, \ldots, n)$, and let $\pi_{i}$ denote the projection of $G$ onto each of the factors; we may assume without loss of generality that each projection $\pi_{i}$ is onto, for otherwise we may replace $H_{i}$ by $\pi_{i}(G)$. Then for each pair $u, v$ of adjacent vertices of $G$ there is a unique index $i$ such that $\pi_{i}$ does not collapse the edge $u v$. This can be visualized within $G$ by coloring the edge $u v$ with color $i$. If this is done for all edges, then we get an $n$-coloring of the edges of $G$. The coloring may be improper: adjacent edges may have the same color. If a 4-cycle $C$ in $G_{i}$ is colored with a single color, then clearly it satisfies the conditions of the lemma. Otherwise, consider the set $E_{C}$ of edges in subgraphs $H_{i}$ onto which the edges of the cycle project. This set of edges must have cardinality two, for $C$ is a subgraph of the product of the edges in $E_{C}$, a hypercube of dimension at most four, but the only 4-cycles in a hypercube are products of two of its factors. In this case, the edges of $C$ have alternating colors, again meeting the conditions of the lemma.

Conversely, suppose that the edges of a median graph $G$ are colored in such a way that every 4-cycle is monochromatic or alternatingly colored. Let $E_{1}, \ldots, E_{n}$ be the partition of the edges of $G$ defined by this coloring. In a median graph $G$, as discussed in Section 2.2 , the cutsets of $G$ consisting of the edges between two complementary half-spaces are the connected components of the "opposite" relation on 4-cycles, and each cutset is therefore monochromatic. In other words, this $n$-coloring is essentially a cutset coloring in the sense of [8, 9], where we do not distinguish between colors corresponding to different edges of the same factor. From this coloring, define $\pi_{i}$ to be a function from $G$ to a graph $H_{i}$ that contracts all edges not belonging to $E_{i}$. Every median graph $G$ is a subgraph of a hypercube, formed by taking the product of a number of copies of $K_{2}$ equal to the number of cutsets of $G$, and $\pi_{i}$ respects this hypercube structure, so $H_{i}$ is a subgraph of a hypercube with dimension $\left|E_{i}\right|$ and $G$ is a subgraph of the product of the graphs $H_{i}$. The median $m(u, v, w)$ of any three vertices in $H_{i}$ may be obtained by finding representatives of $u, v$, and $w$ in $G$ and using $\pi_{i}$ to project the median onto $H_{i}$; therefore, each $H_{i}$ is a median-closed subgraph of a hypercube and therefore is itself a median graph.

Alternatively, for each color $i$ we may define a "congruence" $\psi_{i}$ on the vertex set of $G$ : vertices $x$ and $y$ are congruent modulo $\psi_{i}$ if and only if they are joined by a shortest path whose edges are not colored with color $i$. Then $H_{i}$ is obtained from $G$ by identifying all vertices $x$ and $y$ that are congruent modulo $\psi_{i}$, with two different components being adjacent when connected by an edge from $E_{i}$. Motivated by the algebraic theory of median algebras we say that $G$ has a subdirect representation in terms of the graphs $H_{i} \cong G / \psi_{i}$, in symbols:

$$
G \hookrightarrow \Pi_{i=1}^{n} G / \psi_{i}
$$


Lemma 2. If a median graph $G$ is (improperly) edge-colored so that all of its cutsets are monochromatic, then $G$ has a monochromatic cycle if and only if it has a monochromatic 4-cycle.

Proof. Obviously, if $G$ has a monochromatic 4-cycle then it has a monochromatic cycle. In the other direction, suppose that $C$ is a monochromatic cycle in $G$. Then there is a cutset $\Theta_{1}$ of $G$ containing at least two edges $w_{0} w_{1}$ and $w_{2} w_{3}$ of $C$, where the indices are chosen so that $w_{0}$ and $w_{3}$ are joined by a shortest path $P$ avoiding this cutset. Now any cutset $\Theta_{2}$ of $G$ containing some edge from $P$ must also contain an edge from $C$, thus the edges of this cutset have the same color as the edges of $C$. From the choice of the cutset $\Theta_{2}$ we conclude that the convex splits $\sigma_{1}=\left\{A_{1}, B_{1}\right\}$ and $\sigma_{2}=\left\{A_{2}, B_{2}\right\}$ defined by $\Theta_{1}$ and $\Theta_{2}$, respectively, are incompatible. Notice that in a median graph $G$ two such convex splits are incompatible if and only if there exists a 4-cycle $\left(x_{1}, x_{2}, x_{3}, x_{4}, x_{1}\right)$ of $G$ such that $x_{1} \in A_{1} \cap A_{2}, x_{2} \in B_{1} \cap A_{2}, x_{3} \in B_{1} \cap B_{2}$, and $x_{4} \in A_{1} \cap B_{2}$. Indeed, since the sets $A_{1} \cap A_{2}$ and $B_{1} \cap A_{2}$ are convex and their union is the convex set $A_{2}$, we can find an edge $x y \in \Theta_{1}$ such that $x \in A_{1} \cap A_{2}$ and $y \in B_{1} \cap A_{2}$. Analogously, we can find an edge $x^{\prime} y^{\prime} \in \Theta_{1}$ such that $x^{\prime} \in A_{1} \cap B_{2}$ and $y^{\prime} \in B_{1} \cap B_{2}$. Since the edges $x y$ and $x^{\prime} y^{\prime}$ can be connected by a ladder and $x, y \in A_{2}, x^{\prime}, y^{\prime} \in B_{2}$, necessarily this ladder contain a 4-cycle $\left(x_{1}, x_{2}, x_{3}, x_{4}, x_{1}\right)$ such that $x_{1}, x_{4} \in A_{1}, x_{2}, x_{3} \in B_{1}$ and $x_{1}, x_{2} \in A_{2}, x_{3}, x_{4} \in B_{2}$, establishing our assertion. Since $x_{1} x_{2}, x_{3} x_{4} \in \Theta_{1}$ and $x_{1} x_{4}, x_{2} x_{3} \in \Theta_{2}$ and all edges of the cutsets $\Theta_{1}$ and $\Theta_{2}$ have the same color, we conclude that the 4 -cycle $\left(x_{1}, x_{2}, x_{3}, x_{4}, x_{1}\right)$ is monochromatic.

Now, if all graphs $H_{i}=G / \psi_{i}$ are trees, then there are no monochromatic 4-cycles in $G$. Conversely, if every 4-cycle of $G$ is non-monochromatic and all cutsets of $G$ are monochromatic, then each $H_{i}$ must be a tree. To show this, first notice that any pair of convex splits defined by two cutsets of color $i$ are compatible, or else from the previous assertion we will obtain a monochromatic 4-cycle. Let $A_{1}$ be a minimum by inclusion half-space participating in a convex split $\left\{A_{1}, B_{1}\right\}$ whose cutset $\Theta_{1}$ has color $i$. Then necessarily all edges of the subgraph induced by $A_{1}$ are not colored in color $i$, whence $A_{1}$ is a pendant vertex of the graph $H_{i}$. Employing the induction hypothesis to the coloring of the subgraph of $G$ induced by the convex set $B_{1}$, we conclude that $H_{i}$ is indeed a tree.

Finally, notice that an $n$-coloring of edges of a median graph $G$ such that the opposite edges of each 4-cycle have the same color and the incident edges have different colors is equivalent to the $n$-coloring of the link graph $\operatorname{Link}(|G|)$. In particular, we infer that $G$ is a median subgraph of a Cartesian product of two trees if and only if $\operatorname{Link}(|G|)$ is bipartite.

We summarize the preceding observations in the following result.

Proposition 1. A median graph $G$ is a median subgraph of a Cartesian product of trees $T_{1}, \ldots, T_{n}$ if and only if $G$ admits an n-coloring of its edges such that every $C_{4}$ in $G$ is dichromatic with opposite edges having the same color. The required trees $T_{i}$ are then obtained from $G$ by collapsing all edges colored with a color different from $i$. In particular, a median graph $G$ is a median subgraph of a Cartesian product of two trees if and only if the link graph $\operatorname{Link}(|G|)$ is bipartite. 
This result establishes the equivalence (ii) $\Longleftrightarrow$ (iv) and the implication (ii) $\Longrightarrow$ (vi) of Theorem 11. Indeed, it is easy to see why $\kappa\left(C_{2 k+1}\right)(k \geq 2)$ cannot be embedded in the Cartesian product of only two trees. Since monochromatic 4-cycles are forbidden, consecutive spokes of $\kappa\left(C_{2 k+1}\right)$ (i.e. edges incident with the vertex of degree $2 k+1$ ) have to differ in color, and if only two colors were allowed, they would have to alternate in color. But, as $2 k+1$ is odd, two colors do not suffice. Therefore if a median graph $G$ contains $Q_{3}$ or $\kappa\left(C_{2 k+1}\right)(k \geq 2)$ as an isometric subgraph, then $\tau(G) \geq 3$.

To complete the proof of Theorem 1 1 it remains to establish that (vi) $\Longrightarrow$ (iii). Let $G$ be a median graph. Recall that for every convex split of $G$ there exists at least one edge $x y$ such that $\{W(x, y), W(y, x)\}$ is the given split. Recall also that the half-spaces of the simplex graph $\kappa(F)$ of a graph $F$ are of the form $H_{v}=\{K \in V(\kappa(F)): v \in K\}$ and $V(\kappa(F))-H_{v}$, for each $v \in V(F)[8]$. Hence two convex splits $\left\{H_{v}, V(\kappa(F))-H_{v}\right\}$ and $\left\{H_{w}, V(\kappa(F))-H_{w}\right\}$ are incompatible if and only if $v$ and $w$ are adjacent, that is, $\operatorname{Inc}(\kappa(F)) \cong F$. As we noticed above, two incompatible convex splits of a median graph are associated with at least one 4-cycle $(u, v, w, x, u)$ such that $\{W(u, x), W(x, u)\}$ and $\{W(x, w), W(w, x)\}$ are given splits (where $u x, x w$ are edges of the cycle). Then it is clear that the median graph $G^{\prime}$ obtained from a median graph $G$ by contracting the edges between two complementary half-spaces $W(y, z)$ and $W(z, y)$ has $\operatorname{Inc}(G)-\{W(y, z), W(z, y)\}$ as its incompatibility graph (up to isomorphism).

Lemma 3. Let $G$ be a finite median graph. Assume that for every triple $s_{0}, s_{1}, s_{2} \in$ $V(\operatorname{Inc}(G))$ of compatible (convex) splits there exists an induced path from $s_{1}$ to $s_{2}$ in $\operatorname{Inc}(G)$ which does not contain any neighbor of $s_{0}$ (i.e., any convex split incompatible with $\left.s_{0}\right)$. Then $G$ is a simplex graph such that $G \cong \operatorname{Inc}(\kappa(G))$.

Proof. Suppose there exists a chain $H_{1} \subset H_{0} \subset H_{2}$ of three distinct half-spaces in $G$. Let $H_{i}^{\prime}$ $(i=1, \ldots, k)$ be a sequence of half-spaces such that $H_{1}^{\prime}=H_{1}, H_{k}^{\prime}=H_{2}$, and $\left\{H_{i}^{\prime}, V(G)-H_{i}\right\}$ is incompatible with $\left\{H_{i+1}^{\prime}, V(G)-H_{i+1}^{\prime}\right\}$ and compatible (but distinct) with $\left\{H_{0}, V(G)-\right.$ $\left.H_{0}\right\}(i=1, \ldots, k-1)$. Then, necessarily, either $H_{2}^{\prime}$ or $V(G)-H_{2}^{\prime}$ is properly contained in $H_{0}$ because the corresponding split is compatible with $\left\{H_{0}, V(G)-H_{0}\right\}$ but incompatible with $\left\{H_{1}, V(G)-H_{1}\right\}$. Continuing this way along the given induced path, we eventually conclude that either $H_{k-1}^{\prime}$ or $V(G)-H_{k-1}^{\prime}$ is properly included in $H_{0}$. But then this set is also contained in $H_{2}=H_{k}^{\prime}$, contrary to the incompatibility of the two splits corresponding to $H_{k-1}^{\prime}$ and $H_{2}$. Therefore all chains of half-spaces of $G$ have at most two members. This means that the depth (sensu [9]) of $G$ is at most 2, that is, $G$ is a simplex-graph, i.e., $G \cong \kappa(F)$ and $\operatorname{Inc}(G) \cong \operatorname{Inc}(\kappa(F)) \cong F$, as required. This completes the proof of the lemma.

Now we are in position to conclude the proof of the implication (vi) $\Longrightarrow$ (iii), or equivalently, its contrapositive: a median graph that does not satisfy (iii) also does not satisfy (vi). Thus, assume that $G$ is a median graph the incompatibility graph of which contains an odd cycle (failing to satisfy (iii)) and let $C_{2 k+1}(k \geq 1)$ be a shortest odd cycle in the incompatibility graph $\operatorname{Inc}(G)$ of the convex splits of $G$. We assert that either $G$ is not cube-free, or $G$ contains a convex subgraph isomorphic to $\kappa\left(C_{2 k+1}\right)$, in either case failing (vi). Since the convex hull of 
the 4-cycles in which the incompatible splits of $C_{2 k+1}$ cross is finite, we can assume without loss of generality that the median graph $G$ itself is finite.

Contract successively the edges between complementary half-spaces not belonging to the given $C_{2 k+1}$. This median graph is then isomorphic to $\kappa\left(C_{2 k+1}\right)$ by virtue of the Lemma 3 . Now we reverse the procedure by adding all removed convex splits back one by one, thus performing Mulder's expansion procedure [33, 34]. In the case that $k=1$ the successive contractions produce a cube $Q_{3} \cong \kappa\left(C_{3}\right)$, and in each expansion we retain a copy of this cube. Trivially, a cube subgraph is always convex in a median graph. In the remaining cases, $k \geq 2$; suppose that we have a convex subgraph $\kappa\left(C_{2 k+1}\right)$ at a certain step, as we will be guaranteed to have after all of the contraction steps. Expanding the corresponding larger graph (where $\kappa\left(C_{2 k+1}\right)$ lives in) induces a convex expansion of $\kappa\left(C_{2 k+1}\right)$, possibly a trivial one. We cannot expand along a convex set which contains some $C_{4}$ of $\kappa\left(C_{2 k+1}\right)$, for otherwise, we would get a cube. Therefore the only nontrivial case is attained when we expand only two non-subsequent spokes of $\kappa\left(C_{2 k+1}\right)$. The center $x$ of $\kappa\left(C_{2 k+1}\right)$ then gets copied: $x^{\prime}$ and $x^{\prime \prime}$ are then centers of two simplex graphs of cycles. The number of spokes incident with $x^{\prime}$ plus the number of spokes incident with $x^{\prime \prime}$ equals $(2 k+1)+4$ and hence is again an odd number. So one of the simplex graphs is of the form $\kappa\left(C_{l}\right)$ with $l$ odd number and $l \leq 2 k+1$. By minimality of $k$, we therefore obtain a convex $\kappa\left(C_{2 k+1}\right)$ again in the expanded graph. Thus, $G$ itself must contain either a cube (for $k=1$ ) or a convex $\kappa\left(C_{2 k+1}\right)$ (in the remaining cases), and it fails to satisfy property (vi). This completes the implication from the failure of (iii) to the failure of (vi) and completes the proof of Theorem 1 .

To derive Corollary 1 from Theorem 1 , we establish an auxiliary result which characterizes 2-connectedness for arbitrary median graphs. This immediately yields Corollary 1 , observing that any cutpoint $x$ of $G$ would allow to switch the roles of the two trees in exactly one of the composite subgraphs amalgamated at $x$ in thus obtain an essentially different embedding.

Lemma 4. For a median graph $G$ with at least three vertices, the following conditions are equivalent:

(i) $G$ is 2-connected;

(ii) $\operatorname{Inc}(G)$ is connected;

(iii) $\operatorname{Link}(x)$ is connected for all vertices $x$ of $G$;

(iv) $\operatorname{Link}(G)$ is connected.

Proof. If $G$ is not 2-connected, it is the amalgam of two nontrivial median graphs $G_{1}$ and $G_{2}$ along some cutpoint $x$. Then $\operatorname{Inc}(G), \operatorname{Link}(x)$, and $\operatorname{Link}(G)$ are each the disjoint union of the corresponding graphs of $G_{1}$ and $G_{2}$ and hence disconnected.

If $G$ is 2-connected, then for any incident edges $x y$ and $x z$ of $G$ there exists a path joining $y$ and $z$ that avoids $x$. Choose such a path $P$ with smallest distance sum to $x$. If the maximum distance to $x$ of vertices on $P$ is larger than 2 , then choose any such vertex $v$ and replace it by the median $w$ of $x$ and the two neighbors of $v$ on $P$. Since $v$ and $w$ are at distance 2, one thus obtains a new path still avoiding $x$ but with smaller distance sum to $x$ than $P$, contrary to the choice of $P$. Therefore all vertices on $P$ have distances 1 or 2 to $x$, so that $P$ together 


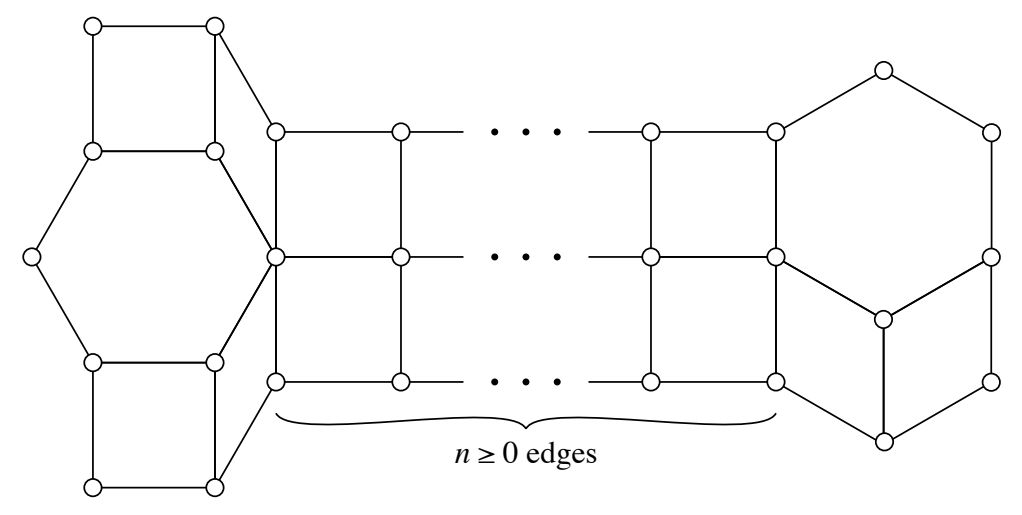

Figure 4. A critical isometric subgraph $H_{n}$ of the Cartesian product of four trees

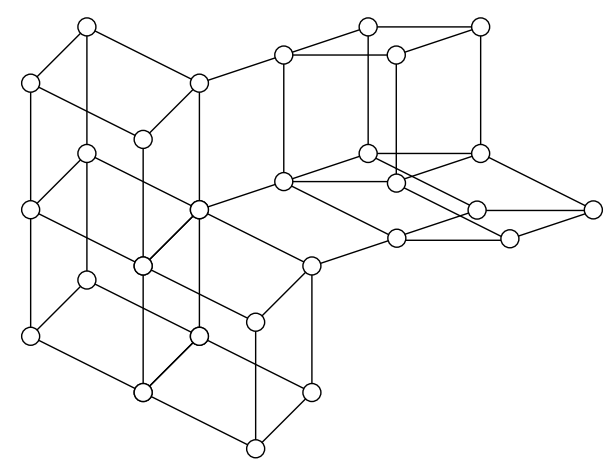

FiguRE 5. The median graph $G$ generated by the graph $H_{1}$ of Fig. 4

with $x$ induces a cogfan in $G$. This cogfan testifies to a path between $x y$ and $x z$ in $\operatorname{Link}(x)$ and to one between the corresponding convex splits in $\operatorname{Inc}(G)$. Considering two non-incident edges $w y$ and $x z$, paths connecting them in $\operatorname{Link}(G)$ and $\operatorname{Inc}(G)$ are provided by virtue of a straightforward induction on the minimal distance, say $d(w, x)$, between the endpoints of $w y$ and $x z$ in $G$.

In Fig. 4 we present an isometric subgraph $H_{n}$ of the Cartesian product of four trees that is critical in the sense that taking any proper isometric subgraph would result in a graph which needs only three trees for an embedding into a Cartesian product of trees. Moreover, collapsing any block of $\Theta$ yields either $H_{n-1}$ (as long as $n>0$ ) or a graph embeddable into a Cartesian product of three trees. The median graph $G$ depicted in Fig. 5 is the median graph generated by the graph $H_{1}$ of Fig. 4 and therefore cannot be embedded into the product of three trees, although every subgraph of $G$ that is a simplex graph needs no more than three trees for an embedding. Thus, this example shows that, in contrast to Theorem 1 , 3-colorability of local neighborhoods does not suffice to characterize isometric subgraphs of Cartesian products of three trees. 


\section{Proof of Theorem 2}

In 7 we described how to recognize squaregraphs in linear time by an algorithm based on breadth-first search. Now we will show that partial double trees also can be recognized in linear time by an algorithm based on lexicographic breadth-first search.

Breadth-First Search (BFS) is one of the simplest algorithmic ways to order the vertices of a connected graph $G$. In BFS, the vertices of the given graph are ordered by their distances from a given base point $b$. The algorithm begins by placing vertex $b$ into an otherwise empty queue. It then repeatedly removes the first vertex $v$ from the queue, and adds to the end of the queue any neighbor $w$ of $v$ that has not already been added to the queue. The neighbor $w$ is referred to as a son of $v$, and conversely $v$ is their father, written in short as $f(w)=v$. When the queue becomes empty, the parent-child relationship between the vertices of $G$ forms a rooted spanning tree $T$ of $G$ with root $b$. Each vertex is inserted and removed once from the queue, and each edge is examined only when one of its endpoints is removed from the queue, so the total time for this procedure is linear in the worst case.

Lexicographic Breadth-First Search (LexBFS), proposed by Rose, Tarjan, and Lueker 16 , 37 , is an alternative procedure for traversing the vertices of a connected graph $G$ in a more specific way: every LexBFS ordering is a BFS ordering, but not necessarily vice-versa. In LexBFS, the queue of vertices is replaced by a queue of sets of vertices, initially containing two sets: the first set in the queue is $\{b\}$ and the second contains all other vertices. In each iteration, the algorithm removes an arbitrarily chosen vertex $v$ from the first set in this queue, and removes the set itself if it becomes empty. When $v$ is removed, the LexBFS algorithm partitions each remaining set $S$ in the queue into two smaller sets, $S \cap N(v)$ and $S \backslash N(v)$; if both of these sets are nonempty, $S$ is removed from the queue and these two sets replace it in the same position, with $S \cap N(v)$ placed earlier in the queue than $S \backslash N(v)$. With some care in the data structures used to represent the queue of sets and to partition the sets, LexBFS can be implemented so that, as with BFS, it takes linear time in the worst case. If one numbers each vertex in the order it is removed, and defines the label $L(x)$ of a vertex $x$ to be the list of its numbered neighbors (already removed from the queue), sorted from smallest to largest, then LexBFS has the property that the sequence of vertex labels it produces is lexicographically sorted so that if $L(y)$ is a proper prefix of $L(x)$, then $x$ is labeled before $y$. Note that this sorting would become the usual (dictionary) lexicographic ordering if we inverted the numbering by starting from $n$ rather than 1 . The parent $f(x)$ of a vertex $x$ is the first vertex in $L(x)$; the vertex with empty label has no parent and is the root $b$ of LexBFS tree of the graph $G$.

Let $G=(V, E)$ be a connected bipartite graph with $n$ vertices. For a total ordering $v_{1}, \ldots, v_{n}$ of vertices of $G$ and an index $1 \leq i \leq n$, we denote by $G_{i}$ the subgraph of $G$ induced by $v_{1}, \ldots, v_{i}$. We will say that $G$ is strongly dismantlable for the given ordering if the following two properties are both satisfied for every vertex $v_{i}$ in $G$ :

(1) If $i>1$, then $v_{i}$ has either one or two neighbors in $G_{i}$.

(2) If $v_{i}$ has two neighbors in $G_{i}$, then $v_{i}$ belongs to a unique 4-cycle of $G_{i}$. 
Lemma 5. If a finite connected bipartite graph $G$ is strongly dismantlable, then the geometric realization $|G|$ is contractible, and therefore, simply connected.

Proof. If $v_{i}$ has only one neighbor $v^{\prime}$ in $G_{i}$, then mapping $v_{i}$ to $v^{\prime}$ yields an elementary contraction from $\left|G_{i}\right|$ to $\left|G_{i-1}\right|$. Otherwise $v_{i}$ belongs to a unique 4-cycle $\left(v_{i}, v^{\prime}, u, v^{\prime \prime}, v_{i}\right)$ of $G_{i}$. Mapping $v_{i}$ to $u$ yields a retraction from $G_{i}$ to $G_{i-1}$ as well as an elementary contraction of the square complex $\left|G_{i}\right|$ to the square complex $\left.\left|G_{i-1}\right|\right|^{1}$ By induction, we conclude that the square complex $|G|$ is contractible.

Observe that, in the LexBFS ordering (or more generally, in any BFS ordering) of any bipartite graph, any 4-cycle abcd must either be ordered with two vertices of the same color first and last (as $a, b, d$, and $c$ ) or with two vertices of one color first and the other color last (as $a, c, b, d)$. We say that the former ordering, $a, b, d, c$, is a proper ordering of the cycle and that the latter ordering, $a, c, b, d$, is an improper ordering.

Lemma 6. If $G$ is a strongly dismantlable graph for a LexBFS ordering of $G$, then all 4-cycles in $G$ are properly ordered by that ordering.

Proof. Suppose to the contrary that a strongly dismantlable graph $G$ contains a 4-cycle $a b c d$ ordered as $a, c, b, d$. For $G$ to be strongly dismantlable, $b$ would have to be part of another 4-cycle abce, with $e$ earlier than $b$ in the ordering. In this case adce would be a 4-cycle containing $d$, as would $a b c d$, contradicting the uniqueness of the 4-cycle containing $d$ among earlier-numbered vertices and therefore contradicting the assumption that $G$ is strongly dismantlable. This contradiction shows that the improperly ordered 4-cycle abcd cannot exist.

Lemma 7. A connected bipartite graph $G$ is strongly dismantlable for a LexBFS ordering of $G$ if and only if

(i) all labels have size at most two,

(ii) for each $x$, if $L(x)=(y, z)$, then $|L(y) \cap L(z)|=1$, and

(iii) no two consecutive vertices in the ordering have equal labels of size two.

Proof. Condition (i) rephrases the condition (1) in the definition of strong dismantlability. Condition (ii) implies that each $v_{i}$ with two neighbors in $G_{i}$ belongs to a unique properly ordered 4-cycle in $G_{i}$, and conditions (i) and (iii) (together with the fact that LexBFS orders all vertices having the same label consecutively) prevent $G$ from containing improperly ordered 4-cycles, so these conditions together imply that each $v_{i}$ with two neighbors in $G_{i}$ belongs to a unique 4-cycle in $G_{i}$. Thus, every graph satisfying the conditions (i)-(iii) of the lemma is strongly dismantlable.

Conversely, suppose that $G$ is strongly dismantlable. For any $v_{i}$ that has two earlier labeled neighbors $y$ and $z$, the unique 4-cycle containing $v_{i}$ in $G_{i}$ must be properly ordered

\footnotetext{
${ }^{1}$ More precisely, according to Whitehead's definition of an elementary contraction in a simplicial complex 40. (p. 247), this step can be represented as a pair of elementary contractions in a triangulation of $|G|$ constructed by splitting each quadrilateral of $|G|$ arbitrarily into two triangles.
} 
by Lemma 6, so its fourth vertex must belong to the labels of both $y$ and $z$, hence (ii) is satisfied. If condition (iii) is not satisfied, then two consecutive vertices in the ordering have equal labels of size two, whence these two vertices together with the vertices of their label form an improperly ordered 4-cycle, violating Lemma 6. Thus, every strongly dismantlable graph meets all the conditions of the lemma.

Lemma 8. Let $G$ be a median graph ordered by LexBFS. If $x$ is any vertex of $G, y$ and $z$ both belong to $L(x)$, and $y \neq z$, then $|L(y) \cap L(z)|=1$.

Proof. Both $y$ and $z$ must be located one step closer from the root $b$ of the LexBFS tree than the vertex $x$, because they have the same color in the bipartition of $G$ and if their distances from $b$ differed by two, then $x$ would come between them in the LexBFS ordering. Let $m$ be the median of $b, y$, and $z$. Since $y$ and $z$ are at distance two apart via a path through $x, m$ must be adjacent to both $y$ and $z$. Thus, $m$ is one step closer to $b$ than $y$ and $z$, so it appears earlier than them in the LexBFS ordering and $m \in L(y) \cap L(z)$. If $L(y) \cap L(z)$ contains a second vertex $m^{\prime}$, then the vertices $m^{\prime}, m, x, y, z$ induce a $K_{2,3}$ subgraph, which is impossible in a median graph.

The next lemma is closely related to Lemma 4 of [7], which states that the farthest vertices from any given vertex in a cube-free median graph have degree at most two, and the proof is essentially the same.

Lemma 9. If $G$ is a cube-free median graph, and $G$ is ordered by LexBFS, then the label of every vertex in $G$ has size at most two.

Proof. Suppose for a contradiction that for some vertex $x, L(x)$ contains three vertices $u$, $v$, and $w$. By Lemma 8 the labels of each pair of these three vertices have a nonempty intersection. If there is a vertex that belongs to all three labels $L(u), L(v)$, and $L(w)$, then that vertex together with $u, v, w$, and $x$ induces a $K_{2,3}$ subgraph, which is impossible in a cube-free median graph. On the other hand, if the three vertices in $L(u) \cap L(v), L(u) \cap L(w)$, and $L(v) \cap L(w)$ are distinct, then these three vertices together with $u, v, w$, and $x$ induce a $Q_{3}-v$ subgraph, again impossible in a cube-free median graph.

Lemma 10. A connected graph $G$ is a partial double tree if and only if

(i) $G$ is strongly dismantlable for the LexBFS ordering, and

(ii) for each vertex $v, \operatorname{Link}(x)$ is bipartite.

Proof. If a connected graph $G$ satisfies the conditions (i) and (ii), then by Lemma 5 it is the underlying graph of a simply connected rectangular complex in which by condition (ii) every $\operatorname{Link}(x)$ is bipartite. Therefore it satisfies condition (iv) of Theorem 4, whence by condition (v) of the same theorem, $G$ is the underlying graph of a partial double tree.

Conversely, suppose that $G$ is a partial double tree. By condition (vi) of Theorem 1, $G$ is a cube-free median graph, in particular $G$ is connected and bipartite and satisfies the conditions of Lemma 8 and Lemma 9. By condition (v) of Theorem 1, it satisfies property (ii) of the lemma. To show that $G$ is strongly dismanlable we verify that each of the three conditions of 
Lemma 7 is met. Condition (i) of Lemma 7 states that each label has at most two vertices; this follows by Lemma 9. Condition (ii) of Lemma 7 must also hold, by Lemma 8. Finally, condition (iii) must also hold, because if two vertices $x$ and $y$ both had equal labels $(u, v)$ then the five vertices in $(L(u) \cap L(v)) \cup\{u, v, x, y\}$ would induce a $K_{2,3}$ subgraph, impossible in a partial double dendron.

Now assume that $G=(V, E)$ is an arbitrary input graph and consider the following simple recognition algorithm:

Step (1): Run the LexBFS algorithm for $G$ and check whether $G$ is connected and bipartite. Test whether each vertex $v_{i}$ has one or two previously labeled neighbors (i.e., $\left|L\left(v_{i}\right)\right| \leq 2$ ), and test whether each two-vertex label $L\left(v_{i}\right)$ is different from $L\left(v_{i-1}\right)$. For each two-vertex label $L\left(v_{i}\right)=(y, z)$, test that $|L(y) \cap L(z)|=1$. Return the answer "No" if $G$ fails to pass any one of these tests.

Step (2): Make a list of 4-cycles of the form $C_{i}=\left(v_{i}, y, z, w\right)$ where $L\left(v_{i}\right)=(y, z)$ and $L(y) \cap L(z)=\{w\}$. Associate with each edge of $G$ a list of the 4-cycles that contain that edge, by initializing an empty list for each edge object and then, for each 4-cycle, adding it to the lists of its four incident edge objects.

Step (3): Using the lists returned by Step (2), for each vertex $v_{i}$ construct $\operatorname{Link}\left(v_{i}\right)$ : the vertices of $\operatorname{Link}\left(v_{i}\right)$ are the edges in $G$ incident to $v_{i}$, and the edges incident to each vertex of $\operatorname{Link}\left(v_{i}\right)$ are given by the 4-cycles incident to the corresponding edge in $G$. Test whether each constructed graph $\operatorname{Link}\left(v_{i}\right)$ is bipartite. If some link is not bipartite, then return the answer "No"; otherwise, return the answer "Yes".

First we show that if the algorithm returns the answer "No", then $G$ is not a partial double dendron. This is obviously true if $G$ is not connected or not bipartite. If the algorithm returns "No" because some vertex has a label that is too large, or because the size of the intersection of two labels is not one, then $G$ fails one of the conditions of Lemma 7 , is not strongly dismantlable, and hence also fails condition (i) of Lemma 10. Finally, if it returns "No" because some vertex has a non-bipartite link, then it fails condition (ii) of Lemma 10. In each case, by Lemma 10, $G$ cannot be a partial double dendron.

Conversely, suppose that the algorithm returns the answer "Yes". Then $G$ must be connected and bipartite. The tests in Step (1) check each condition of Lemma 7, so $G$ must pass all conditions and must be strongly dismantlable, meeting condition (i) of Lemma 10. The test in Step (3) verifies that each link is bipartite, so $G$ meets condition (ii) of Lemma 10. It follows from Lemma 10 that $G$ is a partial double dendron. This concludes the proof of Theorem 2 ,

Note that we are using LexBFS only to ensure that vertices with equal labels are ordered consecutively. A very similar algorithm would work using BFS in place of LexBFS if we modified the test that no two consecutive vertices have equal length-two labels to test instead that no two vertices, consecutive or not, have equal length-two labels. It would also be possible to use an algorithm from [19] in Step (2) to list all 4-cycles in the graph in linear time, but this 
would add unnecessary complexity to the algorithm as the 4-cycles are more easily obtained directly from the labeling information gathered in Step (1). Indeed, if $G$ passes the tests in Step (1), then the list of 4-cycles constituted in Step (2) must include all 4-cycles in $G$, because it includes all properly ordered 4-cycles and because the test that rules out pairs of consecutive equal two-vertex labels eliminates the possibility that an improperly ordered 4-cycle might exist. Finally note that if $G$ is a cube-free median graph ordered by LexBFS, $v_{i}$ is a vertex of $G$ such that $L\left(v_{i}\right)=(y, z)$ and $L(y) \cap L(z)=\{x\}$, then $y$ is the father of $v_{i}$ in the LexBFS tree and $x$ is either the father of both $y$ and $z$ or $x$ is the father of $z[7,14$. This fellow traveller property was used in 7 together with BFS to recognize squaregraphs, namely, to make a list of 4-cycles similar to that constructed in Step (2) of our algorithm.

\section{Proof of Theorem 3}

First we prove the result in the particular case when $\mathcal{K}$ is a square complex. For finite square complexes, the equivalence (i) $\Longleftrightarrow$ (ii) is essentially a particular case of Theorems 3.13 and 3.16 of van de Vel [39, which assert that a finite graph $G$ is median if and only if the cubical complex $|G|$ equipped with the $l_{1}$-metric is a median space. To conclude the proof in the finite case, it remains to notice that the underlying graph of a median square complex $\mathcal{K}$ is cube-free. Suppose by way of contradiction that $G(\mathcal{K})$ contains a 3 -cube $Q_{3}$. Then only the 2-dimensional faces of $Q_{3}$ can correspond to faces of $\mathcal{K}$. Pick the center $u$ of one such face and any two diametral points $v, w$ of the opposite face. The triplet $u, v, w$ contradicts the assumption that $\mathcal{K}$ is median. This shows that the median graph $G(\mathcal{K})$ is cube-free.

To establish the equivalence $(\mathrm{i}) \Longleftrightarrow$ (ii) for infinite square complexes, we will show how to adapt van de Vel's result in this more general framework. First suppose that $G(\mathcal{K})$ is a cube-free median graph. Pick three arbitrary points $x, y, z \in \mathcal{K}$ and three shortest $l_{1}$ paths $\gamma(x, y), \gamma(y, z)$, and $\gamma(z, x)$ in $\mathcal{K}$ between these points. Each of these paths intersects the interior of a finite number of squares of $\mathcal{K}$ (otherwise, we can transform such a path to a path of the same length but containing an infinite number of different vertices of $G(\mathcal{K})$, which is impossible). Consider the convex hull $H$ in $G$ of the vertices of all squares of $\mathcal{K}$ whose interiors intersect $\gamma(x, y) \cup \gamma(y, z) \cup \gamma(z, x)$ and of the edges of $\mathcal{K}$ contained in this union. The set $H$ is finite as a convex hull of a finite set in a median graph [39]. Moreover $H$ is a median subgraph of $G$. By Theorem 3.16 of $[39,|H|$ is a median subspace of $|G|=\mathcal{K}$. Therefore the triplet $x, y, z$ admits a median point in $|H|$, i.e., a point $m^{\prime}$ of $|H|$ lying simultaneously on shortest $l_{1}$ paths $\gamma^{\prime}(x, y), \gamma^{\prime}(y, z)$, and $\gamma^{\prime}(z, x)$ of $|H|$ between $x, y$, and $z$. From the choice of $\gamma(x, y), \gamma(y, z)$, and $\gamma(z, x)$ and the definition of $|H|$ we conclude that $\gamma^{\prime}(x, y), \gamma^{\prime}(y, z)$, and $\gamma^{\prime}(z, x)$ are also shortest $l_{1}$ paths of $\mathcal{K}$, whence $m^{\prime}$ is also a median of $x, y$, and $z$ in $\mathcal{K}$. If $x, y, z$ admit another median point $m^{\prime \prime}$, then pick any shortest $l_{1}$ paths $\gamma^{\prime \prime}(x, y), \gamma^{\prime \prime}(y, z)$, and $\gamma^{\prime \prime}(z, x)$ between $x, y, z$, and passing via $m^{\prime \prime}$. Consider the finite median subgraph $H^{\prime}$ of $G$ defined in the same way as $H$ but with respect to the six paths $\gamma^{\prime}(x, y), \gamma^{\prime}(y, z), \gamma^{\prime}(z, x), \gamma^{\prime \prime}(x, y), \gamma^{\prime \prime}(y, z)$, and $\gamma^{\prime \prime}(z, x)$. Since $m^{\prime}, m^{\prime \prime} \in\left|H^{\prime}\right|, m^{\prime}$ and $m^{\prime \prime}$ are distinct median points of $x, y$, and $z$ in $\left|H^{\prime}\right|$, contrary to the fact that according to Theorem 3.16 of [39], $\left|H^{\prime}\right|$ is a median space. This shows that (i) $\Longrightarrow$ (ii) for arbitrary square complexes. 
To establish that (ii) $\Longrightarrow(\mathrm{i})$, let $\mathcal{K}$ be a square complex such that the metric space $(\mathcal{K}, d)$ is median. First we show that $G(\mathcal{K})$ is an isometric subspace of $(\mathcal{K}, d)$. Pick two vertices $x, y$ of $G(\mathcal{K})$ and a shortest $l_{1}$ path $\gamma(x, y)$ in $\mathcal{K}$. We will transform $\gamma(x, y)$ into a path of $G(\mathcal{K})$ with the same length. We can assume without loss of generality that $\gamma(x, y)$ does not pass via any vertex $z$ of $G(\mathcal{K})$, otherwise we can apply our argument separately to the pairs $\{x, z\}$ and $\{z, y\}$. If $\gamma(x, y)$ crosses two incident sides of a square $S$ of $\mathcal{K}$, then replacing the subpath of $\gamma(x, y)$ contained in $S$ by the subpath (of the same length) between the intersection points of $\gamma(x, y)$ with the sides of $S$ and going instead via the common vertex of these sides, we will obtain a shortest $l_{1}$ path $\gamma^{\prime}(x, y)$ passing via a vertex of $G(\mathcal{K})$, to which we can apply the argument above. Therefore we can suppose that if $\gamma(x, y)$ intersects a square of $\mathcal{K}$, then it crosses its boundary in two opposite sides. As $x$ and $y$ are vertices, this is possible only if $\gamma(x, y)$ is a path of the graph $G(\mathcal{K})$. Hence $G(\mathcal{K})$ is an isometric subspace of $(\mathcal{K}, d)$. Each square and each edge of $\mathcal{K}$ are compact convex subsets of $(\mathcal{K}, d)$, therefore they are gated sets 39]. We assert that the gate of each vertex $v$ of $G(\mathcal{K})$ in this set is also a vertex of the underlying graph. We will show this for squares $S$ of $\mathcal{K}$, the proof for edges is analogous. Let $v^{\prime}$ be the gate of $v$ in $S$ and suppose that $v^{\prime}$ is an inner point of the edge $[a, b]$ of $S$. Let $S^{\prime}$ be the square of $\mathcal{K}$ sharing with $S$ the side $[a, b]$ and intersecting some shortest $l_{1}$ path between $v$ and $v^{\prime}$. If the gate of $v$ in $S^{\prime}$ belongs to a side incident to $[a, b]$, then necessarily the gate of $v$ in $S$ will be one of the vertices $a$ or $b$ and not $v^{\prime}$. So, assume that the gate of $v$ in $S^{\prime}$ belongs to the interior of the side of $S^{\prime}$ opposite to $[a, b]$. Continuing the same reasoning with $S^{\prime}$ instead of $S$, we will construct a sequence squares, such that the gates of $v$ in two consecutive squares of this sequence belong to the interior of the opposite sides of the first square. This sequence is finite because each time the distance from $v$ to the respective gate decreases by at least by 1 . This is obviously impossible, because the last square of the sequence has $v$ as a vertex. Hence, indeed the gate of $v$ in each face or edge of $\mathcal{K}$ is a vertex. To complete the proof, it remains to show that the median $m$ in $\mathcal{K}$ of any three vertices $x, y, z$ of $G(\mathcal{K})$ is also a vertex of this graph. Suppose by way of contradiction that $m$ is an inner point of some square $S$ of $\mathcal{K}$ (the case when $z$ belongs to the interior of an edge is analogous). The gates $x^{\prime}, y^{\prime}, z^{\prime}$ of $x, y, z$ in $S$ are all vertices of $S$. Since $m$ belong to the interval between $x$ and $y$, necessarily there exists a shortest $l_{1}$ path between $x$ and $y$ traversing $x^{\prime}, m$, and $y^{\prime}$ in this order. This is possible only if $x^{\prime}$ and $y^{\prime}$ are opposite corners of $S$. Analogously, we deduce that $x^{\prime}, z^{\prime}$ and $y^{\prime}, z$ are also opposite corners of $S$. Since this is impossible, necessarily $m$ is a vertex of $G(\mathcal{K})$. As $G(\mathcal{K})$ is an isometric subspace of the median space $(\mathcal{K}, d)$, we conclude that $G(\mathcal{K})$ is a median graph. This establishes the implication (ii) $\Longrightarrow(\mathrm{i})$.

The equivalence $(\mathrm{i}) \Longleftrightarrow$ (iii) in the case of square complexes is a particular case of a result of 14,36 which establishes that the underlying graph of a cubical complex $\mathcal{K}$ is median if and only if $\mathcal{K}$ equipped with the $l_{2}$-metric is $\operatorname{CAT}(0)$. On the other hand, Gromov 25 characterized cubical $\mathrm{CAT}(0)$ complexes in the following pretty manner: A cubical complex $\mathcal{K}$ is $\mathrm{CAT}(0)$ if and only if $\mathcal{K}$ is simply connected and satisfies the following condition: whenever three $(k+2)$-cubes of $\mathcal{K}$ share a common $k$-cube and pairwise share common $(k+1)$-cubes, they are contained in $a(k+3)$-cube of $\mathcal{K}$. Applying this characterization to square complexes 
$\mathcal{K}$, the combinatorial condition is equivalent to the assertion that $\mathcal{K}$ does not contain three squares which share a common vertex and pairwise share common edges, i.e., that $\mathcal{K}$ does not contain a vertex $x \in V(\mathcal{K})$ containing a $C_{3}$ in $\operatorname{Link}(x)$. In view of previous equivalences, this shows that (iv) is equivalent to the first three conditions of Theorem 3 .

It remains to establish the result for rectangular complexes $\mathcal{K}$. Define a linear map between each rectangular face $R$ of $\mathcal{K}$ and the respective face of the square complex $|G(\mathcal{K})|$. One can easily show that the image of any $l_{1}$ path of $\mathcal{K}$ under the resulting piecewise linear map is an $l_{1}$ path of $|G(\mathcal{K})|$, and vice versa, the preimage of any $l_{1}$ path of $|G(\mathcal{K})|$ is an $l_{1}$ path of $\mathcal{K}$. Therefore, the equivalence $(\mathrm{i}) \Longleftrightarrow(\mathrm{ii})$ follows from a similar result for square complexes established above. On the other hand, the equivalence (iii) $\Longleftrightarrow$ (iv) is a direct consequence of another result of Gromov [13,25] characterizing the polygonal complexes with $\mathrm{CAT}(0) l_{2}$-metrics. According to this result, a rectangular complex $\mathcal{K}$ with a finite number of isometry types of cells is $\mathrm{CAT}(0)$ if and only if $\mathcal{K}$ does not contain a vertex $x \in V(\mathcal{K})$ with a $C_{3}$ in $\operatorname{Link}(x)$. To establish the equivalence between the conditions (i),(ii) and the last two conditions (iii),(iv) notice that, in view of the result of [14,36] mentioned above, the underlying networks of rectangular complexes satisfying these conditions are the same: they are median and cube-free. Finally, if any of the conditions of Theorem 3 holds, then $\mathcal{K}$ equipped with the intrinsic $l_{1}$-metric $d$ is median and, by Theorem $3.13(3)$ of [39] and its extension to arbitrary rectangular complexes, $\mathcal{K}$ coincides with the geometric realization $|N(\mathcal{K})|$ of its network. This concludes the proof of Theorem 3.

\section{Proof of Theorem 4}

To establish the implication (i) $\Longrightarrow$ (ii) suppose that the rectangular complex $\mathcal{K}$ is isometrically embedded into the Cartesian product $D=D_{1} \times D_{2}$ of two dendrons $D_{1}$ and $D_{2}$. We wish to show that the median $m$ of any three points $x, y$, and $z$ of $\mathcal{K}$ taken in this double dendron must belong to $\mathcal{K}$. Note that for any given finite set $F$ of points in $\mathcal{K}$ we can let the projections $F_{1}$ and $F_{2}$ of $F$ onto $D_{1}$ and $D_{2}$ subdivide the line segments, so that $F_{1}$ and $F_{2}$ may be taken as subsets of the vertex sets of $D_{1}$ and $D_{2}$, respectively. In particular, let $F$ be the set of all vertices of $\mathcal{K}$ together with $x, y$, and $z$. Therefore the underlying network $N(\mathcal{K})$ of the complex $\mathcal{K}$ is isometrically embedded into the double tree network $N(D)$. Every shortest path in the network $N(D)$ is also a shortest path in the double tree $G(D)$, and vice versa. In particular, the isometric embedding between $N(\mathcal{K})$ and $N(D)$ is also one between the underlying graphs $G(\mathcal{K})$ and $G(D)$. Therefore $G(\mathcal{K})$ is a partial double tree and thus a median graph by Theorem 3 . Consequently, the median $m$ of $x, y$, and $z$ in $G(\mathcal{K})$ also serves as the corresponding median in the underlying double tree $G(D)$ and hence in $D$.

First note that (ii) $\Longleftrightarrow$ (iii) holds by virtue of Theorem 3 . To show that (iii) $\Longrightarrow$ (iv), suppose by way of contradiction that for some vertex $x \in V(\mathcal{K}), \operatorname{Link}(x)$ contains an odd cycle of length $2 k+1$ with $k \geq 1$. This means that we can find $2 k+1$ squares $R_{1}, \ldots, R_{2 k+1}$ of $\mathcal{K}$, all sharing the vertex $x$ and constituting an odd rectangular wheel. Denote by $x x_{i}^{\prime}$ the common edge of the rectangles $R_{i}$ and $R_{i+1(\bmod 2 k+1)}$. Now, let $B(x, \epsilon)$ be the closed neighborhood with radius $\epsilon>0$ centered at $x$ which is a partial double dendron. On each segment $I\left(x, x_{i}^{\prime}\right)$ 
pick a point $x_{i}$ located at the same distance $\delta<\epsilon$ from $x$ such that for each $i$ the square $\left(x, x_{i}, y_{i}, x_{i+1(\bmod 2 k+1)}\right)$ contained in the rectangle $R_{i}$ also belongs to the closed ball $B(x, \epsilon)$. Since inside each rectangle $R_{i}$ the distance is measured according to $l_{1}$, we conclude that $\left(x, x_{i}, y_{i}, x_{i+1(\bmod 2 k+1)}\right)$ is a rectangle of the metric space $(\mathcal{K}, d)$ (and thus of $\left.(B(x, \epsilon), d)\right)$. As a consequence, we derived an odd rectangular wheel in the partial double dendron $B(x, \epsilon)$, which is impossible. This establishes that (iii) $\Longrightarrow($ iv).

To show that (iv) $\Longrightarrow(\mathrm{v})$, first notice that since $\mathcal{K}$ is simply connected and the links of all vertices are $C_{3}$-free, Theorem 3 implies that $G(\mathcal{K})$ is a cube-free median graph. Since each $\operatorname{Link}(x)$ is bipartite, the graph $G(\mathcal{K})$ does not contain odd cogwheels, thus from Theorem 1 we infer that $G(\mathcal{K})$ is a partial double tree. The equality $\mathcal{K}=|G(\mathcal{K})|$ follows from last assertion of Theorem 3 .

Then, to show that $(\mathrm{v}) \Longrightarrow(\mathrm{i})$, notice that if $G(\mathcal{K})$ embeds isometrically into the Cartesian product of two trees $T_{1}$ and $T_{2}$, then all edges of $G(\mathcal{K})$ from the same cutset color-class have the same length in the rectangular complex $\mathcal{K}$. Now, transform each tree $T_{i}$ into a dendron $D_{i}$ having the same topology as $T_{i}$ and obtained by replacing every edge of $T_{i}$ by a solid edge of $D_{i}$ of length equal to the length of the edges of $\mathcal{K}$ from the respective color class. This leads to a natural mapping $\varphi$ from $\mathcal{K}$ to $D=D_{1} \times D_{2}$. It can be checked in a standard way that this mapping is an isometric embedding of $\mathcal{K}$ into $D=D_{1} \times D_{2}$.

As for the implication $(\mathrm{v}) \Longrightarrow(\mathrm{vi})$, if $G=G(\mathcal{K})$ is a partial double tree, then $G$ can be regarded as a median subgraph of two trees $T_{1}$ and $T_{2}$ such that the projection from $G$ to either tree is surjective (Theorem 1). Then both trees are finite as $\mathcal{K}$ is finite. The pre-image $T$ of any leaf $t$ of $T_{2}$ under the projection from $G$ to $T_{2}$ is a finite tree, the neighbors in $G$ of which form an isomorphic copy $U$ projected to the neighbor $u$ of $t$. Then $G$ can be recovered as the convex expansion of $G-T$ along $U$ 33. If $x$ is a vertex of $U$ having two neighbors $y$ and $z$ in $U$ such that $x$ is the hub of some $k$-cogfan $H$ in $G-T$ where $y$ and $z$ have degree 2 in $H$, then the convex expansion extends $H$ to a $(k+2)$-cogwheel in $G$. Hence $k$ is necessarily even by Theorem 11. Evidently, $\mathcal{K}=|G|$ is the convex expansion of $|G-T|$ along the finite dendron $|U|$ by the interval $[0, \lambda]$, where $\lambda$ is the weight of the edges between the subnetworks $G-T$ and $T$. Note that any gated dendron in $\mathcal{K}$ is a finite dendron which can be regarded as the geometric realization of some finite tree in the network of some refinement of the complex $\mathcal{K}$. Therefore a straightforward induction establishes $(\mathrm{v}) \Longrightarrow(\mathrm{vi})$.

The implication (vi) $\Longrightarrow$ (vii) is trivial because gated expansions are particular instances of gated amalagamations. Finally, to prove the implication (vii) $\Longrightarrow$ (iv), proceed by a trivial induction on the number of amalgamation steps. Since amalgamation of median rectangular complexes $\mathcal{K}_{1}$ and $\mathcal{K}_{2}$ along gated sets evidently yields a median space, condition (ii) of Theorem 3 holds, whence $\mathcal{K}$ is simply connected by Theorem 3 . The links of vertices of $\mathcal{K}$ which do not belong to the dendron $A$ along which the last amalgamation was performed are as in the respective constituent and hence bipartite according to the induction hypothesis. $\operatorname{Link}_{1}(x)$ and $\operatorname{Link}_{2}(x)$ of a vertex $x$ on $A$ in the two gated constituents $\mathcal{K}_{1}$ and $\mathcal{K}_{2}$ are glued along the vertices representing the edges on $A$. Since all paths in $\operatorname{Link}_{1}(x)$ and $\operatorname{Link}_{2}(x)$ 

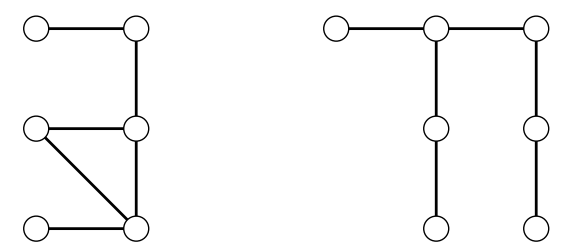

FIGURE 6. A six-vertex asymmetric graph and a seven-vertex asymmetric tree

connecting any pair of the latter vertices have the same parity, no odd circle arises and therefore $\operatorname{Link}(x)$ is bipartite, too. This completes the proof of Theorem 4 .

\section{Proof of Theorem 5}

By Frucht's Theorem 22] any group can be represented as the automorphism group of some graph $F$ (which can be chosen to be finite if the group is finite). We stipulate that the trivial group is represented by a nontrivial graph, say, by an asymmetric graph with six vertices or an asymmetric tree with seven vertices (Fig. 6). Since every automorphism maps components to components, we may add an asymmetric tree with seven vertices in the case that the graph did not yet have this tree as a component.

Next we claim that $F$ and its simplex graph $\kappa(F)$ have isomorphic automorphism groups. Clearly every automorphism $f$ of $F$ maps simplices to simplices of the same cardinality and trivially preserves inclusion between simplices. Therefore $f$ lifts to the simplex map $\kappa f$ which is an automorphism on $\kappa(F)$. Conversely, every automorphism of $\kappa(F)$ that fixes the empty simplex is lifted from some automorphism of $F$. Since $F$ has a component with a convex 3 -path, all maximal hypercubes in $\kappa(F)$ intersect in a single vertex, viz. the empty simplex node. This property guarantees that every automorphism of $\kappa(F)$ fixes the latter vertex, because maximal hypercubes are mapped onto maximal hypercubes by automorphisms of $\kappa(F)$.

Since $\kappa(F)$ is a non-singleton median graph, the iterated simplex graph $G=\kappa(\kappa(F))$ is 2 -connected and has radius 2. The automorphism groups of $\kappa(F)$ and $\kappa(\kappa(F))$ are isomorphic because the intersection of all maximal hypercubes (4-cycles) in $\kappa(\kappa(F))$ is the empty simplex node of $\kappa(\kappa(F))$ (as $\kappa(F)$ includes some 4-cycle). The incompatibility graph $\operatorname{Inc}(\kappa(\kappa(F)))$ of the convex splits of $\kappa(\kappa(F))$ is isomorphic to the bipartite graph $\kappa(F)$, whence $\kappa(\kappa(F))$ is a partial double tree by Theorem 1 .

It remains to prove the last assertion of Theorem 5, that aut $(G)=\operatorname{aut}(|G|)$. Clearly, every automorphism of $G$ extends to an automorphism of $|G|$; we must show that $|G|$ has no other automorphisms than the ones constructed in this way. To do so, we show that $G$ can be determined uniquely as a graph from the metric structure of $|G|$, without starting from any knowledge of the decomposition of $|G|$ into cells. However, this is straightforward: because of the construction of $G$ as a cube-free simplex graph, the 2-cells of $G$ are exactly the subsets that are isometric to $l_{1}$ unit squares and that have the additional property that at least two consecutive sides of the square consist of boundary points (points with a neighborhood 
homeomorphic to a half-plane whose boundary passes through the point). Because $G$ is a simplex graph of a graph without isolated vertices, every vertex or edge of $G$ is incident to a 2-cell in $|G|$, and the edges and vertices of $G$ are then exactly the sides and corners of these squares. Alternatively, one could also recognize the underlying graph $G$ here by the two observations that first the degree 2 vertices $u_{i}(i \in I)$ of $G$ are exactly the points $p$ of $|G|$ for which $|G|-\{p\}$ is a median space and second every vertex $x$ of $G$ is recognized via the requirement that $d\left(x, u_{i}\right)+d\left(x, u_{j}\right)-d\left(u_{i}, u_{j}\right)$ be an even integer for all $i, j \in I$. This concludes the proof of Theorem 5

\section{REFERENCES}

1. A. Aho, J. Hopcroft, and J. Ullman, On finding lowest common ancestors in trees, Proc. 5th ACM Symp. Theory of Computing (STOC), 1973, pp. 253-265.

2. S. Alstrup, C. Gavoille, Haim Kaplan, and T. Rauhe, Nearest Common Ancestors: A Survey and a New Algorithm for a Distributed Environment, Theory Comput. Syst. 37 (2004), no. 3, 441-456.

3. S.P. Avann, Metric ternary distributive semi-lattices, Proc. Amer. Math. Soc. 12 (1961), 407-414.

4. H.-J. Bandelt, Networks with Condorcet solutions, Europ. J. Oper. Res. 20 (1985), 314-326.

5. _ Hereditary modular graphs, Combinatorica 8 (1988), 149-157.

6. H.-J. Bandelt and V. Chepoi, Metric graph theory and geometry: a survey, Surveys on Discrete and Computational Geometry: Twenty Years Later (J. E. Goodman, J. Pach, and R. Pollack, eds.), Contemp. Math., vol. 453, AMS, Providence, RI, 2008, pp. 49-86.

7. H.-J. Bandelt, V. Chepoi, and D. Eppstein, Combinatorics and geometry of finite and infinite squaregraphs, Electronic preprint arxiv:0905.4537, 2009.

8. H.-J. Bandelt and M. van de Vel, Embedding topological median algebras in products of dendrons, Proc. London Math. Soc. (3) 58 (1989), 439-453.

9. __ Superextensions and the depth of median graphs, J. Combin. Th. Ser. A 57 (1991), 187-202.

10. G. Birkhoff and S. A. Kiss, A ternary operation in distributive lattices, Bull. Amer. Math. Soc. 52 (1947), 749-752.

11. L. M. Blumenthal, Theory and Applications of Distance Geometry, Clarendon Press, Oxford, 1953.

12. B. H. Bowditch, Treelike Structures Arising from Continua and Convergence Groups, Mem. Amer. Math. Soc., vol. 662, 1999.

13. M. Bridson and A. Haefliger, Metric Spaces of Non-Positive Curvature, Springer-Verlag, 1999.

14. V. Chepoi, Graphs of some CAT(0) complexes, Adv. Appl. Math. 24 (2000), 125-179.

15. V. Chepoi, F. Dragan, and Y. Vaxès, Center and diameter problem in planar quadrangulations and triangulations, Proc. 13th Annu. ACM-SIAM Symp. on Discrete Algorithms (SODA 2002), 2002, pp. 346-355.

16. D. G. Corneil, Lexicographic breadth first search - a survey, Graph-Theoretic Methods in Computer Science, Lecture Notes in Computer Science, vol. 3353, Springer-Verlag, 2004, pp. 1-19.

17. M. de Berg, O. Cheong, M. van Kreveld, and M. Overmars, Computational Geometry: Algorithms and Applications, 3rd ed., Springer-Verlag, 2008.

18. A. Dress and R. Scharlau, Gated sets in metric spaces, Aequationes Math. 34 (1987), 112-120.

19. D. Eppstein, Arboricity and bipartite subgraph listing algorithms, Inform. Process. Lett. 51 (1994), no. 4, $207-211$.

20. __ Optimally fast incremental Manhattan plane embedding and planar tight span construction, Electronic preprint arxiv:0909.1866, 2009.

21. D. Eppstein, J.-Cl. Falmagne, and S. Ovchinnikov, Media Theory, Springer-Verlag, 2007.

22. R. Frucht, Herstellung von Graphen mit vorgegebener abstrakter Gruppe., Compos. Math. 6 (1938), 239250 . 
23. E. Ghys and P. de la Harpe, Les Groupes Hyperboliques d'après M. Gromov, Progress in Mathematics, vol. 83, Birkhäuser, 1990.

24. R. L. Graham and P. M. Winkler, On isometric embeddings of graphs, Trans. Amer. Math. Soc. 288 (1985), 527-536.

25. M. Gromov, Hyperbolic groups, Essays in Group Theory (S. M. Gersten, ed.), MSRI Publications, vol. 8, Springer-Verlag, 1987, pp. 75-263.

26. D. Harel and R. E. Tarjan, Fast algorithms for finding nearest common ancestors, SIAM J. Comput. 13 (1984), no. 2, 338-355.

27. W. Imrich and S. Klavžar, Product Graphs: Structure and Recognition, Wiley Interscience, New York, 2000 .

28. W. Imrich, S. Klavžar, and H. M. Mulder, Median graphs and triangle-free graphs, SIAM J. Discr. Math. 12 (1999), no. 1, 111-118.

29. J. R. Isbell, Median algebra, Trans. Amer. Math. Soc. 260 (1980), 319-362.

30. S. Klavžar and M. Kovše, Induced cycles in crossing graphs of median graphs, submitted, 2008.

31. K. Menger, Untersuchungen über allgemeine Metrik, I-III, Math. Ann. 100 (1928), 75-163.

32. J. S. B. Mitchell, Geometric shortest paths and network optimization, Handbook of Computational Geometry (J.-R. Sack and J. Urrutia, eds.), Elsevier, Amsterdam, 2000, pp. 633-701.

33. H. M. Mulder, The structure of median graphs, Discr. Math. 24 (1978), 197-204.

34. _ The Interval Function of a Graph, Math. Centre Tracts, vol. 132, Mathematisch Centrum, Amsterdam, 1980.

35. A. Papadopulos, Metric Spaces, Convexity andNonpositive Curvature, IRMA Lectures in Mathematics and Theoretical Physics, vol. 6, European Mathematical Society, 2005.

36. M. Roller, Poc sets, median algebras and group actions, Univ. of Southampton, preprint, 1998.

37. D. J. Rose, R. E. Tarjan, and G. S. Lueker, Algorithmic aspects of vertex elimination on graphs, SIAM J. Comput. 5 (1976), no. 2, 266-283.

38. M. van de Vel, Matching binary convexities, Topology Appl. 16 (1983), 207-235.

39. Theory of Convex Structures, Elsevier, Amsterdam, 1993.

40. J. H. C. Whitehead, Simplicial spaces, nuclei and m-groups, Proc. London Math. Soc. (2) 45 (1939), 243-327.

Dept. of Mathematics, University of Hamburg, Bundesstr. 55, D-20146 Hamburg, Germany

E-mail address: bandelt@math.uni-hamburg.de

Laboratoire d'Informatique Fondamentale, Université d' Aix-Marseille, Faculté des Sciences de Luminy, F-13288 Marseille Cedex 9, France

E-mail address: chepoi@lif.univ-mrs.fr

Computer Science Department, University of California, Irvine, Irvine CA 92697-3435, USA

E-mail address: eppstein@ics.uci.edu 